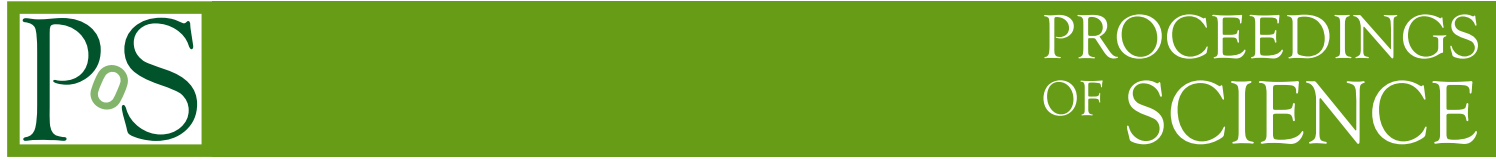

\title{
Rooted staggered fermions: good, bad or ugly?
}

\section{Stephen R. Sharpe*}

Department of Physics, University of Washington, Seattle, WA 98195-1560, USA

E-mail: sharpe@phys.washington.edu

I give a status report on the validity of the so-called "fourth-root trick", i.e. the procedure of representing the determinant for a single fermion by the fourth root of the staggered fermion determinant. This has been used by the MILC collaboration to create a large ensemble of lattices using which many quantities of physical interest have been and are being calculated. It is also used extensively in studies of QCD thermodynamics. The main question is whether the theory so defined has the correct continuum limit. There has been significant recent progress towards answering this question. After recalling the issue, and putting it into a broader context of results from statistical mechanics, I critically review the new work. I also address the related issue of the impact of treating valence and sea quarks differently in rooted simulations, discuss whether rooted simulations at finite temperature and density are subject to additional concerns, and briefly update results for quark masses using the MILC configurations. An answer to the question in the title is proposed in the summary.

XXIVth International Symposium on Lattice Field Theory

July 23-28, 2006

Tucson, Arizona, USA

\footnotetext{
${ }^{*}$ Speaker.
} 


\section{Introduction}

The field of lattice QCD is at a strange juncture in its history. A significant fraction of the community is using an approach ("rooted staggered fermions"- to be explained in detail below) which others in the community feel rests on weak theoretical foundations, and yet others think may be wrong. There is a lot at stake because simulations using rooted staggered fermions have been computationally faster than those with other fermions (although improvements in algorithms may have closed this gap) and are already able to make accurate calculations of a number of physical quantities. Results for "gold plated" masses and form factors (i.e. those which can be calculated most straightforwardly, with systematic errors controlled) agree with experimental results within the claimed few percent accuracy $[1,2,3,4,5]$, and several successful predictions have been made (as summarized in Ref. [6]). Rooted staggered lattices are presently being used for many "mixed action" calculations involving valence quarks with a different action, most notably domainwall fermions with an almost exact chiral symmetry. Applications to light and heavy-quark flavor physics and nucleon physics are described in other talks at this meeting [7, 8, 9].

Thus, in the spirit of a well-known western, we need to know, and the broader particle and nuclear physics communities need to know, whether the results using rooted staggered fermions are

- GOOD, i.e. have the correct continuum limit, without any complications;

- BAD, i.e. have the wrong continuum limit; or

- UGLY, i.e. have the correct continuum limit, but with unphysical contributions present for $a \neq 0$ ( $a$ being the lattice spacing), requiring theoretical understanding and complicated fits.

Let me stress that, if the continuum limit is wrong, then the results from rooted simulations are simply wrong, and there is no a priori reason for them to be close to those of QCD. In particular, it would make no sense to try and estimate systematic errors due to rooting. The situation differs from that with the quenched approximation (or the much better approximation of using $N_{f}=2$ light dynamical flavors while quenching the strange quark) where one does expect many results to be close to those of QCD. Of course, it is always possible that rooted simulations are "bad" but give results numerically close to those of QCD for some quantities-in other words, that the numerical successes to date are a fluke. It is because of this possibility, and because it is hard to make a convincing argument by numerical results alone, that I focus on theoretical arguments in this talk. For more discussion of numerical tests see last year's review by Dürr [10], and the recent work of Refs. [11, 12, 13, 14].

During the last year, there has been significant theoretical progress towards understanding whether rooting is valid, with issues clarified and in some cases resolved. There are, however, no quick and easy answers. Many of the relevant papers are long and detailed, and this appears to be unavoidable given the technical nature of the issue. The result is that, although I have attempted to give only summaries, and pick out the essential points, this article is itself long and somewhat technical. Because of this, I provide a thorough overview of the organization of the article in the following, so as to allow a reader to skip to the core sections if so desired. 
I begin in the next section by collecting the (uncontroversial) general assumptions that I make. I then briefly recall the definition and general properties of staggered fermions (sec. 3), with some details on perturbation theory relegated to app. A, and explain what "rooting" means and why it is used (sec. 4). This brings me in sec. 5 to the first discussion of new work, namely the argument of Bernard, Golterman and Shamir that rooted staggered fermions cannot be described by a local single-taste theory except in the continuum limit [15]. The main problem faced by rooting is now identified: non-locality.

At this point I switch gears, and give in sec. 6 an update on the status of the MILC ensemble of rooted staggered lattice configurations, and the results obtained with them that are not discussed in other plenary talks at this meeting.

Returning to my main theme, sec. 7 discusses what we learn about non-local theories from studies of lower-dimensional statistical mechanics systems. The subsequent section, sec. 8, is the core of this talk, where I describe the arguments which have been given to understand and "tame" the non-locality. These use perturbation theory (PT), renormalization group (RG) methods (the work of Shamir [16]), and chiral perturbation theory ( $\chi$ PT) (the work of Bernard [17]). I also discuss the numerical evidence concerning the $\beta$-function with rooted staggered fermions.

In sec. 9 I describe some potentially serious problems with rooted staggered fermions which have been raised by Creutz [18, 19] and, I think, answered by Bernard, Golterman, Shamir and myself [20] and by Dürr and Hoelbling [21]. The residue of these concerns is a clearer understanding of the ugly features of rooted staggered fermions. A different concern (the "valence rooting issue") is discussed in sec. 10, with most of the details of its resolution collected in app. B.

Most of the work in the last year has argued for the correctness of rooting. An important exception is the study of non-zero density where Golterman, Shamir and Svetitsky have observed that there is an additional problem [22]. I discuss this in sec. 11, as well as the status of rooting in simulations at non-zero temperature.

I summarize and give my conclusion-good, bad or ugly? —in sec. 12.

A reader wishing to focus on developments on the central issue can skip secs. 2, 6, 7, 9, 10 and 11, as well as the appendices. Someone familiar with staggered fermions and the meaning of rooting can also skip secs. 3 and 4. Put the other way, the key sections are 5, 8, and the conclusions.

\section{Assumptions}

In this talk I will assume several results that, while not proven, are generally accepted. For the sake of clarity and completeness I collect them here.

The first assumption is the foundation of the field: that lattice QCD with any standard choice of fermion other than staggered (i.e. Wilson-like [including clover and twisted mass], overlap and domain-wall) has a universal, non-trivial continuum limit. In other words, I assume that these "uncontroversial" fermion discretizations are "good", in my classification above. There is no rigorous proof of this, but it is strongly supported by perturbation theory (asymptotic freedom plus scaling of irrelevant operators) and extensive numerical, non-perturbative tests. The best that one could hope for in a discussion of rooted staggered fermions would be to show that they have the same continuum limit as that of one of the uncontroversial fermions. 
The second assumption is that staggered fermions without the rooting trick (which I will refer to by the ungainly but descriptive term "unrooted") are uncontroversial, and have a continuum limit with four degenerate fermions, conventionally called "tastes". ${ }^{1}$ The arguments for this are on a slightly weaker theoretical footing than with Wilson-like fermions, but are nonetheless strong, as I discuss in passing below.

My final working assumption will be that the violations of reflection positivity that usually accompany the use of improved fermion (or gauge) actions are unimportant for physical quantities. In other words, while they give rise to unphysical degrees of freedom (e.g. with complex energies or negative metric), these are restricted to the cut-off scale. I mention this point because essentially all staggered simulations use improved actions (as do simulations with other fermion actions).

\section{What are staggered fermions?}

Euclidean staggered fermions have a very simple and economical action. In unimproved form ${ }^{2}$ this is [23]

$$
\bar{\chi} D_{\text {stag }} \chi=\sum_{n} \bar{\chi}_{n}\left[\sum_{\mu} \frac{\eta_{n, \mu}}{2}\left(U_{n, \mu} \chi_{n+\mu}-U_{n-\mu, \mu}^{\dagger} \chi_{n-\mu}\right)+m_{0} \chi_{n}\right],
$$

where $n$ labels sites in a 4 dimensional lattice, $\chi_{n}$ are one-component fermions, $U_{n, \mu}$ are the usual gauge links and $m_{0}$ is the bare lattice mass (in lattice units, so $m_{0}=a m_{\text {cont }}$ ). The Dirac matrices are cleverly packaged into the position dependent signs $\eta_{n, \mu}$, whose form I will not need.

This is the action that is simulated, ${ }^{2}$ with numerical efficiency resulting from the single component nature of the fermions (as compared to the usual four Dirac components). But this action does not make manifest the physical degrees of freedom. It suffers from the doubling problem, having $2^{4}=16$ one-component fermions in the classical continuum limit. These can be packaged into 4 tastes of 4 -component Dirac fermions. One way to do this is to divide the lattice into $2^{4}$ hypercubes, labeled by $n_{y}$ (with all components even), with the sites inside each hypercube labeled by a "hypercube vector" $B$ with binary components $[24,25]$. In the free theory, the $16 \chi$ 's in each hypercube are packaged into a Dirac-taste matrix as follows:

$$
Q_{\beta, b}\left(n_{y}\right)=\frac{1}{8} \sum_{B}\left[\gamma_{B}\right]_{\beta, b} \chi_{n_{y}+B} ; \quad \gamma_{B}=\gamma_{1}^{B_{1}} \gamma_{2}^{B_{2}} \gamma_{3}^{B_{3}} \gamma_{4}^{B_{4}}
$$

The free staggered action [eq. (3.1) with $U_{n, \mu}=1$ ] can be exactly rewritten as follows (using momentum space for later convenience):

$$
\sum_{p_{y}} \bar{Q}\left(p_{y}\right)\{\underbrace{\left[\sum_{\mu} i\left(\gamma_{\mu} \otimes \mathbf{1}\right) \sin p_{y, \mu}+(\mathbf{1} \otimes \mathbf{1}) m_{0}\right]}_{O(a)}+\underbrace{\sum_{\mu}\left(\gamma_{5} \otimes \xi_{\mu} \xi_{5}\right)\left(1-\cos p_{y, \mu}\right)}_{O\left(a^{2}\right)}\} Q\left(p_{y}\right) .
$$

\footnotetext{
${ }^{1}$ Tastes were, in the past, referred to as "staggered flavors" or simply "flavors". "Tastes" is preferred since, in the standard usage described below, the four tastes are reduced to one (by rooting), and flavor is introduced as an additional degree of freedom.

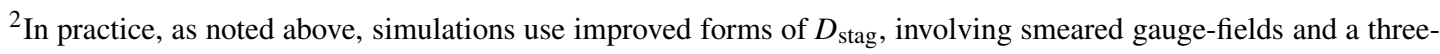
step (or knight's move) derivative. These improvements do not impact the following considerations, so I do not show them explicitly.
} 
Here $p_{y}$ is the momentum (in lattice units) conjugate to the hypercube vector $n_{y}$, and so does not include oscillations within the hypercubes. Such high momenta correspond to the doubler modes on the original lattice and are here included as degrees of freedom in $Q$ itself. The notation $\left(\gamma_{B} \otimes \xi_{C}\right)$, which will recur repeatedly in this talk, indicates the matrices acting on the Dirac $\left(\gamma_{B}\right)$ and taste $\left(\xi_{B}\right)$ indices of $Q_{\beta, b}$, namely $\beta$ and $b$ respectively. The taste generators $\xi_{B}$ are defined like $\gamma_{B}$ in (3.2), but with $\xi_{\mu}=\gamma_{\mu}^{\star}$. (The complex conjugation here is a convenient, but not fundamental, convention.) The $\xi_{B}$ form a basis for the generators of the taste group $S U$ (4).

In the form (3.3), the (free) staggered action looks like four Wilson fermions with an unconventional Wilson term. If the Dirac-taste matrix in the Wilson term were replaced by $(\mathbf{1} \otimes \mathbf{1})$ then one would simply have four Wilson fermions. With the form shown, however, the Wilson term both removes the doubler modes (those with $p_{y, \mu} \approx \pi$ ) from the continuum spectrum and breaks most of the taste symmetries (both vector and axial). What is gained by breaking these taste symmetries is that one axial generator remains unbroken by the Wilson term, that which generates the so-called $U(1)_{\varepsilon}$ symmetry:

$$
Q \rightarrow \exp \left[i \alpha\left(\gamma_{5} \otimes \xi_{5}\right)\right], \quad \bar{Q} \rightarrow \bar{Q} \exp \left[i \alpha\left(\gamma_{5} \otimes \xi_{5}\right)\right]
$$

This symmetry remains in the interacting theory, where it must be defined at the level of the $\chi$ fields. The situation should be contrasted with standard Wilson fermions, where the Wilson term breaks all the axial but none of the vector symmetries. The importance of the $U(1)_{\varepsilon}$ symmetry is that, unlike with standard Wilson fermions, there is no renormalization of the critical mass - it remains at $m_{0}=0$. This is one of the practical advantages of staggered fermions.

As indicated by the underbraces in eq. (3.3), the Wilson term is irrelevant in the continuum limit when $p \sim O(a)$. One's naive expectation is thus that the $S U(4)$ taste symmetry is restored as $a \rightarrow 0$, just as the broken axial symmetries are restored with normal Wilson fermions. This argument is naive because, in the interacting theory, the packaging transformation (3.2) must include gauge fields to retain gauge invariance, and the gauge fields living "inside" the $Q$ 's can also lead to taste breaking. A careful analysis must enumerate possible relevant operators consistent with the symmetries of the original lattice action. Such an analysis [26] finds no additional relevant operators, which, coupled with asymptotic freedom, implies that the symmetry restoration in the free theory applies also in the presence of interactions. This is the basis for my assumption that "unrooted" staggered fermions have the expected continuum limit.

The description so far uses what is called, for obvious reasons, the "position-space basis"spin and taste are distributed over a space-time hypercube. For many applications, and in particular for perturbative calculations, it is more convenient to use a different basis, one set up in momentum space. This is described in app. A.

\section{Rooted staggered fermions}

We do not want a continuum theory with four degenerate quarks - we want QCD with nondegenerate quarks. The obvious approach is to somehow make the tastes non-degenerate in the continuum limit, so that they could correspond to the $u, d, s$ and $c$ quarks. This can be done by adding additional terms to the action (involving $\chi$ and $\bar{\chi}$ on different, but nearby, sites) [27, 28, 26]. Furthermore, the coefficients of each of these terms is multiplicatively renormalized $[29,30,31$, 
26], i.e. the lattice symmetries remain strong enough to forbid mixing with the identity operator. This approach has not, however, been followed in practice, and it is perhaps worthwhile discussing why.

I think this is for four main reasons. First, there is a tuning problem. The physical masses are linear combinations of the coefficients which are multiplicatively renormalized. Since we only know these renormalizations approximately (e.g. in one-loop perturbation theory), the coefficients would have to be tuned numerically to obtain the correct physical masses. Perhaps more important is that, although one can pick mass terms such that the determinant is real, it is not necessarily positive, and its spectrum has a general complex form. This is compared to the real, positive determinant of staggered fermions with its spectrum lying on a line (parallel to the imaginary axis) and bounded away from the origin. Thus at least part of the numerical advantage of staggered fermions would likely be lost. Third, since the general masses break some of the lattice symmetries, continuum multiplets fragment into even smaller lattice representations. And, finally, the action would be complicated to simulate.

What is done in practice is to use one staggered fermion for each flavor and to take the fourthroot of the fermion determinant: ${ }^{3}$

$$
Z_{\mathrm{QCD}}^{\mathrm{root}}=\int D U e^{-S_{\text {gauge }}}\left\{\operatorname{det}\left[D_{\text {stag }}\left(m_{u}\right)\right] \operatorname{det}\left[D_{\text {stag }}\left(m_{d}\right)\right] \operatorname{det}\left[D_{\text {stag }}\left(m_{s}\right)\right]\right\}^{\mathbf{1 / 4}},
$$

where I have restricted myself to the three light flavors. ${ }^{4}$ Note that $\operatorname{det}\left(D_{\text {stag }}\right)$ is positive definite for any non-zero quark mass (eigenvalues come in pairs due to the $U(1)_{\varepsilon}$ symmetry: $\pm i \lambda+m_{0}$ with $\lambda$ real), and one always takes the positive fourth-root.

The rationale for "rooting" is that, in the continuum limit, each $D_{\text {stag }}$ represents four degenerate tastes, and thus has the structure $D_{1} \otimes \mathbf{1}$, where $D_{1}$ is a one taste operator. If $\operatorname{so,} \operatorname{det}\left[D_{\text {stag }}\right]=$ $\operatorname{det}^{4}\left(D_{1}\right)$, and rooting is legitimate. For $a \neq 0$, however, taste is broken and this argument fails. We thus arrive at the central question addressed in this talk:

\section{Is it legitimate to take the fourth-root of the fermion determi- nant before sending $a \rightarrow 0$ ?}

\section{Non-locality of single-taste action and its implications}

The basic problem with rooting has been clear from the beginning: locality. The determinant of a local operator can be written as a fermionic functional integral with a local action - this is how the determinant arises in the first place. Locality is one of the properties required of the action to ensure that the underlying theory is physical at long distances. ${ }^{5}$ The fourth-root of the determinant of a general local operator without an $S U$ (4) symmetry cannot, however, be written in any obvious way as a functional integral over a fermion with a local action. It can certainly be written as a functional integral, e.g. with the action $\bar{\chi}_{\text {bad }}\left(D_{\text {stag }}\right)^{1 / 4} \chi_{\text {bad }}$ in the present case, but the action here

\footnotetext{
${ }^{3}$ This method was introduced to study the Schwinger model in Ref. [32].

${ }^{4}$ Most simulations to date work in the isospin symmetric limit with $m_{u}=m_{d}$, so the two fourth-roots combine to a square-root in the up-down sector.

${ }^{5}$ See the review by Jansen [33] for a thorough discussion of all the properties that are needed and their status with different fermion discretizations.
} 
is non-local (even in the free theory) [34]. The correct question is whether there is a clever way of manipulating $D_{\text {stag }}$ to arrive at a local fermionic formulation. The answer is now clear from the work of Bernard, Golterman and Shamir (BGS) [15]: NO. In particular,

\section{Rooted staggered fermions cannot be described by a local the- ory with a single taste per flavor.}

The argument for this is straightforward, and proceeds by contradiction. The most general way to represent the rooted determinant by a local single-taste theory requires

$$
\left(\operatorname{det}\left[D_{\text {stag }}\right]\right)^{1 / 4}=\operatorname{det}\left[D_{1}\right] \exp \left(-\delta S_{\text {eff,g }}\right) \text {, }
$$

where $D_{1}$ a local, single-taste operator and $\delta S_{\text {eff,g }}$ is a local gauge action. The latter is allowed because $\delta S_{\text {eff,g }}$ can be absorbed into the gauge action [35,36]. The result $(5.1)$ is assumed to hold on all configurations, a condition which might be relaxed, as discussed below. It follows that the fermionic weight for "unrooted" staggered fermions is

$$
\operatorname{det}\left[D_{\text {stag }}\right]=\left(\operatorname{det}\left[D_{1}\right]\right)^{4} \exp \left(-4 \delta S_{\text {eff,g }}\right)=\operatorname{det}\left(D_{1} \otimes \mathbf{1}\right) \exp \left(-4 \delta S_{\text {eff, g }}\right) .
$$

In words, this result implies that the unrooted staggered theory can be rewritten as a local theory with an exact $S U(4)$ taste symmetry. But we know that this is false. The staggered action retains only a discrete subgroup of the $S U(4)$ taste symmetry, and thus its spectrum will not fall into $S U(4)$ multiplets. In particular, while the lightest states in the $S U$ (4) symmetric theory will be a 15-plet of pseudo-Goldstone bosons (PGBs), the staggered theory has only a single PGB (associated with the $\left.U(1)_{\varepsilon}\right)$, with the other 14 light pseudoscalars falling into multiplets of the lattice symmetry with masses differing from the PGB by $O\left(a^{2}\right)$. For an example of a concrete contradiction consider the connected part of the two-point correlator of the plaquette. At long distances this will be dominated by two-pseudoscalar contributions, and there is clearly no way that these can match over a range of distances given the different masses of the states in the two theories.

Thus we learn that the starting assumption, eq. (5.1), is false: a local one-taste formulation is not possible. Any one-taste formulation of rooted staggered fermions must be non-local. A nonlocal action can lead to a resolution of the contradiction because the notion of a spectrum (needed in the argument above) can no longer be used. An explicit example of such a resolution will be seen in sec. 8.3 [see eq. (8.7) and subsequent discussion].

An obvious corollary of the BGS result is that, if rooted staggered fermions are to yield a physical continuum limit describing a single taste, the non-locality must vanish when $a \rightarrow 0$. How this can occur is the focus of sec. 8 below. I only note here that it is consistent with eq. (5.2), because the spectrum on the 1.h.s. does become $S U(4)$ symmetric in the continuum limit (given the assumption that the unrooted staggered theory is "good"), and so a local $\delta S_{\text {eff,g }}$ suffices.

The BGS argument is not completely watertight. The relation (5.1) needs to hold only on the subset of configurations that are important in the rooted ensemble, and it could be that this subset has no overlap with that which is important in the unrooted ensemble. This would invalidate the necessity of non-locality. I am not sure how plausible such a loophole is, but in any case I do not pursue this possibility because the RG and $\chi \mathrm{PT}$ arguments in sec. 8 imply that rooted staggered fermions are indeed non-local for $a \neq 0$, consistent with the conclusion of the BGS argument. 
Rooted staggered fermions have reached their nadir at this point in the talk. A reasonable person might say:

"Locality of the action guarantees universality, i.e. that we obtain the correct continuum limit. Non-local theories are unphysical (lacking unitarity, ...). The possibility that the non-locality vanishes in the continuum limit sounds hard to establish, even implausible. I don't want to use rooted staggered fermions."

This is not, I think, the only reasonable reaction. A second reasonable person might say:

"Rooted staggered fermions are so attractive numerically, that I am going to try and understand and "tame" the non-locality. If I can argue plausibly that the non-locality does not change the universality class, i.e. that all the effects of non-locality vanish in the continuum limit, then the extensive numerical results based on the MILC ensemble will be physical, as long as the chiral and continuum extrapolations take into account the effects of non-locality."

Most of the rest of the talk will concern the attempts to tame the non-locality. First, however, I want to recall why the stakes are so high, and at the same time briefly change roles into a reviewer of the status of staggered simulations. ${ }^{6}$

\section{Update on rooted staggered simulations}

In this section I will set aside the theoretical questions about rooting, and review numerical progress with rooted staggered simulations.

Over the last 5 or so years, the MILC collaboration has created a large ensemble of configurations using the rooting trick with a particular variant of improved staggered fermions (the "asqtad" action $-a^{2}$ terms removed at tree-level from both fermion and gauge actions, and tadpole improved [38]). Aside from the concerns about rooting, this ensemble has been very successful and is a model for future ensembles using other fermion discretizations. The most important features are:

1. All three light quarks are unquenched, i.e. these are fully unquenched simulations. In practice, " $2+1$ " flavors are used, with $m_{\ell}=m_{u}=m_{d}$, so that isospin is exact. This is a good enough approximation for few percent accuracy in many physical quantities.

2. The light quarks are truly light, ranging down to $m_{\ell} \approx m_{s}^{\text {phys }} / 10$. Having good statistics at such low masses is crucial for accurate chiral extrapolations.

3. There are large numbers (of order 500) of independent lattice configurations for each value of the parameters, so that statistical errors in many quantities are smaller than systematic errors.

4. The lattices are widely available, so that many quantities of physical interest can and have been calculated on the ensemble by many collaborations. This feature of the MILC ensemble

\footnotetext{
${ }^{6}$ For an update on the status with other fermions see the talk by Giusti [37].
} 
has set a precedent that is happily being followed quite widely, facilitated by the ILDG (International Lattice Data Grid), as reviewed here by Jansen [39].

Until recently, one of the weakness of the MILC ensemble was that it lacked sufficient lattice spacings to allow a thorough test of the continuum extrapolation (particularly important in light of the non-locality issue). Previously there were "fine" lattices with $a \approx 0.09 \mathrm{fm}$ and "coarse" lattices with $a \approx 0.12 \mathrm{fm}$. Now there are also "medium coarse" ( $a \approx 0.15 \mathrm{fm})$ and the beginnings of a "super-fine" ( $a \approx 0.06 \mathrm{fm})$ set. The latter is clearly the most important and will be filled out substantially this year. There are also "very coarse" ( $a \approx 0.18 \mathrm{fm})$ lattices, but for many quantities the taste-breaking may be too large for these lattices to be useful. A complete list of available lattices is available in the contribution of Sugar [40].

What remains as a shortcoming is that only in one case is it possible to make a comparison between two different volumes. This is not a criticism of the MILC collaboration-they have simply had to make hard choices given the large computational resources required. The problem is well illustrated by the size of the super-fine lattices: $48^{3} \times 144$ and up!

As noted above, these lattices are being used for a wide range of physics applications. These are discussed in the plenary talks on flavor physics [7, 8] and hadronic physics [9]. It is clearly crucial, however, that the systematics of taste-breaking are numerically well understood in simple quantities if we are to trust the results for more complicated applications. To illustrate the status I show in Fig. 1 results for the ratio $m_{\pi}^{2} /\left(m_{x}+m_{y}\right)$ versus the sum of the valence quark masses $m_{x}+m_{y}$ [40]. Almost all the points are partially quenched (PQ), with the upper cluster (circles) being on the medium-coarse lattices, the middle cluster (diamonds and crosses) from the coarse lattices, and the lower cluster (squares) from the fine lattices. The lines through the points are part of a global fit to $m_{\pi}, m_{K}, f_{\pi}$ and $f_{K}$, a generalization of that described in Ref. [3]. The fits are to (rooted) staggered $\chi \mathrm{PT}$, a theory discussed below, and have good confidence levels. The predicted extrapolation to the continuum limit, including an interpolation to the physical strange quark mass, is shown as the (red) line at the bottom. The vertical line at about 0.008 on the horizontal axis shows the resulting physical average light quark mass. I stress that the continuum limit result shown in Fig. 1 is not PQ - it is for a pion composed of degenerate sea quarks of mass $m_{x}=m_{y}=m_{\ell}$.

The main point I want to make with this plot is that the fit allows one to predict where the data from the single super-fine lattice should lie. This prediction is shown by the dotted (blue) line, along with the new data (dotted fancy pluses). Note that (in contrast to the continuum prediction discussed above) this is a prediction for a PQ pion (sea quark masses held fixed and valence masses $m_{x}$ and $m_{y}$ varying). The predicted curvature is an example of an enhanced PQ logarithm [41]. The prediction works well (remembering that all these PQ points are correlated).

I draw two conclusions, one staggered-specific and one general. First, PGB properties from rooted staggered simulations can be well described using staggered $\chi$ PT. This numerical test could have failed, as illustrated by complete failure of fits to continuum $\chi \mathrm{PT}$ [3]. Second, this is one example of a growing body of evidence that PQ $\chi$ PT can successfully describe PQ simulations (irrespective of the type of fermions). This observation will be useful below.

The one physics result that I want to quote is an update on values for quark masses:

$$
\begin{aligned}
m_{s}^{\overline{\mathrm{MS}}}(2 \mathrm{GeV})=90(0)(5)(4)(0) \mathrm{MeV} & {[87(0)(4)(4)(0) \mathrm{MeV}] } \\
m_{s} / \widehat{m}=27.2(0)(4)(0)(0) & {[27.4(1)(4)(0)(1)] }
\end{aligned}
$$




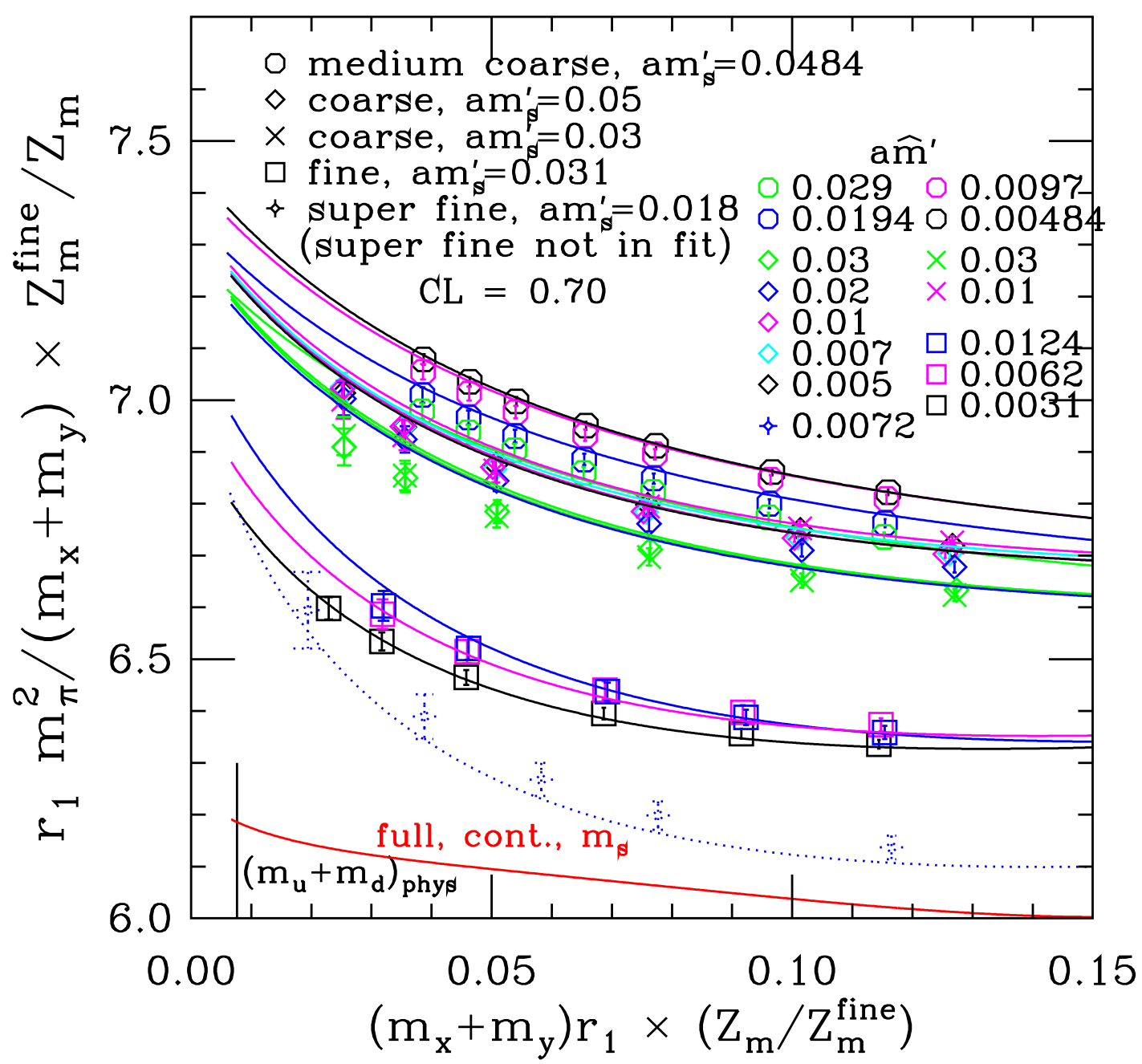

Figure 1: Update on pion mass results using rooted improved staggered fermions. The scale is set with $r_{1}$. $Z$-factors account for logarithmic scaling of quark masses. See text for further explanation.

$$
m_{u} / m_{d}=0.42(0)(1)(0)(4) \quad[0.43(0)(1)(0)(8)] .
$$

The new results (still preliminary) include the super-fine and medium-coarse lattices [40], and should be compared to the old results [shown in parentheses] which used only the coarse and fine lattices [2, 3, 42]. The four errors are from (i) statistics, (ii) fitting and other systematics of the simulations, (iii) perturbation theory, and (iv) the uncertainty in the estimates of EM effects. The point I want to emphasize here is that the inclusion of the first super-fine lattice changes the results at a level consistent with the quoted errors. It is clearly important to see whether this remains true as the super-fine ensemble is extended.

I choose this result as it is an example of a (potentially) successful completion of one of the key goals of lattice simulations: determination of the fundamental parameters of QCD. Knowing $m_{s}$ to better than $10 \%$, and ruling out $m_{u}=0$ at the $10-\sigma$ level, are impressive results. But are they correct? One issue is the theoretical concern about rooting - the focus of the rest of my talk. Aside from this, there is the more mundane question of whether the two-loop perturbative matching [42] 
that is employed is sufficiently accurate. The inclusion of the two-loop contributions raised $m_{s}$ by $14 \%$ compared to using one-loop matching, and the estimated three-loop error $\left( \pm 2 \alpha_{s}^{3} \approx \pm 4 \mathrm{MeV}\right.$ in $m_{s}$ ) is a guess, albeit a reasonable one. It is clearly important to check the matching factor using non-perturbative renormalization-calculations are underway, and we should know by next year.

To date, there is no fully unquenched result with "uncontroversial" fermions to which this rooted staggered result can be compared. Nevertheless, there are interesting results with $N_{f}=2$ flavors of improved Wilson fermions that suggest important lessons. In particular, they indicate that using non-perturbative renormalization can change the result by an amount larger than the naive guess of the size of higher-order perturbative corrections. For example, Ref. [43] finds that, at a lattice spacing corresponding to the fine MILC lattices, $m_{s}$ is $24 \%$ higher with the non-perturbative $\mathrm{Z}$ factor than it would be with the one-loop value.

A second lesson concerns the lattice spacing dependence. The simulations of Ref. [43] have pushed to finer lattices than MILC, and find a significant dependence on $a^{2}$ between the fine MILC spacing and the continuum limit. While there is no particular reason that the same should be true with (rooted) staggered fermions, this underlines the importance of completing the super-fine ensemble and possibly pushing to yet finer lattices.

As is well known, rooted staggered fermions have been used to calculate a number of "goldplated" quantities, finding agreement with experiment at the few percent level [1]. Furthermore, predictions for the $D \rightarrow K \ell v$ form factor, $f_{D}$ and the $B_{c}$ mass were subsequently confirmed by experiment (as summarized in Ref. [6]). While these successes are impressive, numerical checks cannot be definitive. For one thing, the required fitting is complicated, as illustrated by Fig. 1. We would want calculations with other fermion discretizations even without the rooting issue. It could also be that the agreement is a fluke-a wrong theory could give results close to experiment for some quantities but not in general. But the most important reason that numerical tests do not suffice is that there is a serious theoretical problem (non-locality), and it must be understood.

\section{Non-local interactions in statistical mechanics}

I now take a brief excursion into statistical mechanics (SM) to see what lessons can be learned about non-local interactions. In particular, while for local interactions we expect the universality class to be determined by the number of dimensions and by the symmetries of the order parameter, this will not be true in general with non-local interactions.

There is a considerable literature studying power-law interactions in two and three dimensions, motivated in part by physical interactions such as the van de Waals force. One well-studied example is the Ising-like model in $d$-dimensions with Hamiltonian:

$$
H=\mathscr{J} \sum_{\vec{x}, \vec{y}} s_{\vec{x}} “ \frac{1}{|x-y|^{d+\sigma}} " s_{\vec{y}} .
$$

The quotes around the power-law indicate that a smoothed non-singular form is used, which has no impact on the long distance behavior. $\mathscr{J}$ is the coupling, and $\sigma$, a positive real number, characterizes the non-locality. The non-locality increases as $\sigma$ is reduced.

Such theories have been studied using all the tools of SM and I quote some examples. The original works used renormalization group (RG) methods and the $\varepsilon$-expansion [44, 45], with recent extensions studying corrections to scaling, e.g. Ref. [46]. There are a number of exact results, 


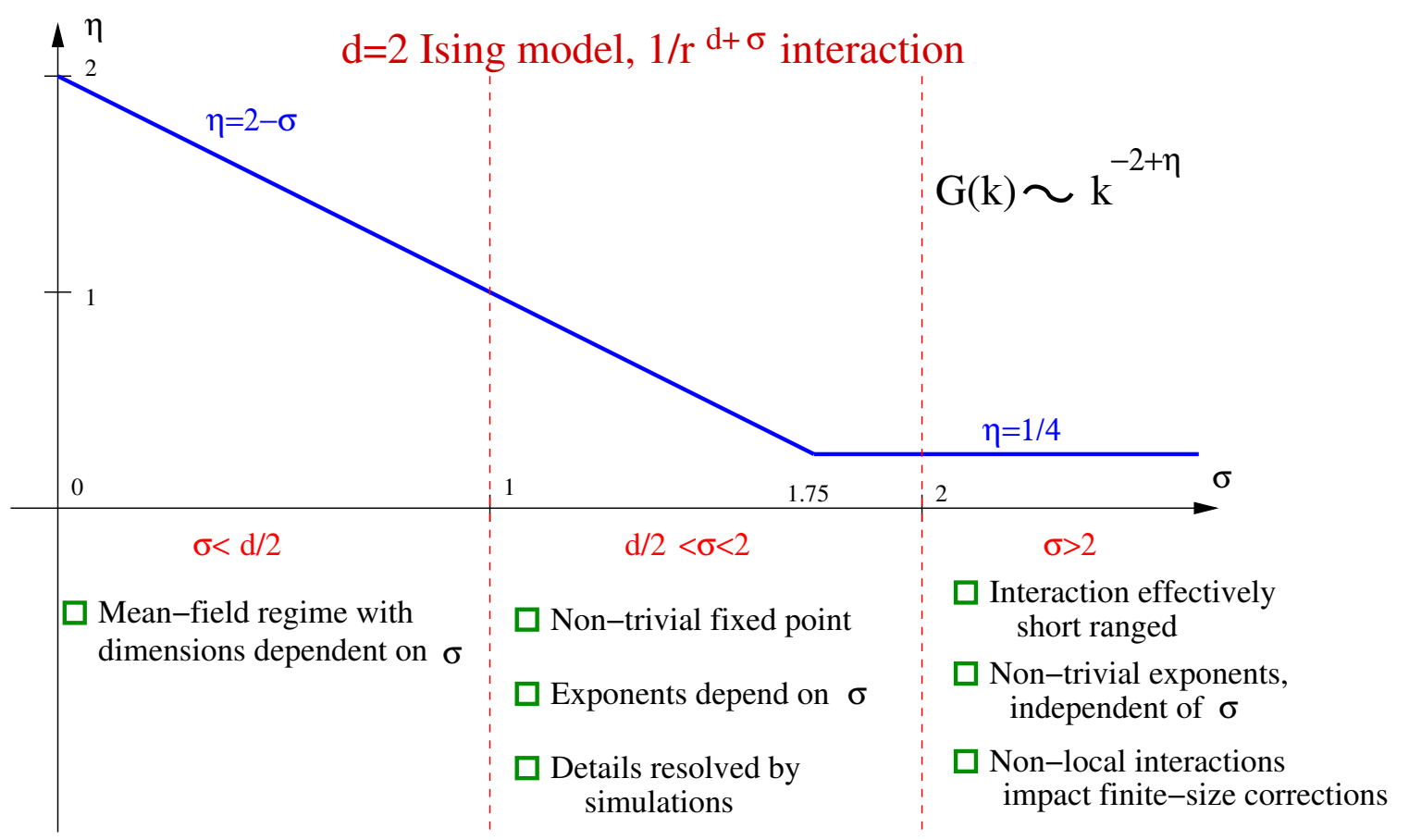

Figure 2: Results for correlation-length exponent for $d=2$ Ising model with long-range interactions.

e.g. Ref. [47]. There have also been numerical simulations, e.g. Refs. [48, 49], which, as can be imagined, are challenging for non-local interactions. I do not have time or competence to discuss all this work, and note only one simple point. In the RG analysis, after blocking, the spins are replaced by scalar fields, which have a $\phi^{4}$ interaction, and a bare propagator whose inverse at long distances has the form:

$$
G_{0}(k)^{-1} \sim r+j_{\sigma} k^{\sigma}+j_{2} k^{2}+\ldots
$$

with $r, j_{\sigma}$ and $j_{2}$ calculable constants. This is obtained by Fourier transforming the interaction in (7.1), and holds as written for non-integer $\sigma$ (otherwise there are logarithmic corrections). This expression shows that in the free theory, when $r$ is tuned to its critical value of zero (by varying the temperature), the IR behavior is dominated by the non-local part of the interaction if $\sigma<2$, but, conversely, the non-local part is sub-dominant if $\sigma>2$.

These observations help one understand the results that have been obtained for a variety of models with non-local interactions. I have chosen the $d=2$ Ising-like model as a concrete example and collect in Fig. 2 results for the critical exponent $\eta$ (taken from Ref. [49] and references therein). Recall that $\eta$ is defined by the small $k$ dependence of the full propagator at the critical point: $G(k) \sim k^{-2+\eta}$. What one learns from an RG analysis is that, as suggested above, for $\sigma>2$ the interaction falls off fast enough that it is effectively short ranged, and one finds the usual local critical exponents (here $\eta=1 / 4$ ). The key point is that these are independent of $\sigma$. It should be noted, however, that the non-local interactions do impact finite-volume corrections. For example, in the disordered phase above the critical temperature, and assuming periodic boundary conditions, finite-volume corrections are exponentially suppressed with local interactions (as are corrections to hadron masses in QCD), but are power-law suppressed, as $L^{-(d+\sigma)}$, with non-local 
interactions [46].

For $\sigma<2$, i.e. more slowly falling interactions, the $k^{\sigma}$ term in the free propagator dominates in the IR, changing the scaling analysis. If $\sigma<d / 2$ one finds that the $\phi^{4}$ interaction is irrelevant, leading to mean-field-like behavior, with $G(k) \sim k^{-\sigma}$ at criticality. Thus one finds $\eta=2-\sigma$, so that the critical exponent does depend on $\sigma$. Crudely speaking, a non-local interaction increases the effective number of neighbors, which is the same effect as occurs when one increases the dimension, and one is effectively pushed above the upper critical dimension (which is 4 for local interactions) into the mean-field regime. Finally, for $d / 2<\sigma<2$ the $\phi^{4}$ interaction is relevant and the critical behavior non-trivial. The exponents can be estimated using the $\varepsilon$-expansion, and calculated using simulations. They depend, in general, on $\sigma$, as in the example shown in the Fig. 2.

Clearly one must be careful in drawing lessons from these results. For one thing, rooted staggered QCD has a dominant interaction, that from the gauge sector, which is local. For another, we are at the upper critical dimension, and so corrections to scaling are logarithmic rather than power law (which corresponds to the middle regime, $d / 2<\sigma<2$, being squeezed to a point). Nevertheless, I draw the following four general lessons:

- Non-local interactions need not change the universality class (the $\sigma>2$ case above). Thus there is no a priori reason to rule out rooted staggered fermions. Instead, one must study the issue.

- Perturbation theory (which is the analog of the RG equations in the SM examples) is a useful tool for such a study.

- One should check the perturbative conclusions using simulations. A crucial question is whether the scaling is as in the desired continuum limit, i.e. whether $\alpha_{s}$ runs correctly.

- One should beware of enhanced finite size effects—standard $\chi$ PT predictions for such effects will likely fail. As we will see, this can be remedied by using "rooted" staggered $\chi$ PT.

While we do not really need the SM example to come to these conclusions, I find that it helps "demystify" the issue of non-locality.

\section{Can we tame the non-locality?}

I now come to the core of this talk. We know that QCD with rooted staggered fermions involves non-local interactions, and the key question is whether this non-locality changes the continuum limit. I will discuss in turn what has been learned using perturbation theory, numerical simulations, a general RG analysis, and $\chi \mathrm{PT}$.

\subsection{Perturbation theory}

In this section I discuss what we learn about QCD with rooted staggered fermions using perturbation theory. I go into some detail because, although some of the discussion has been given in the literature, I am unaware of a complete account.

I start from the assumed renormalizability of lattice QCD with unrooted staggered fermions. That is, by adding the usual (finite number of) counterterms, which depend logarithmically on $a$, 
one finds that properly normalized correlation functions with non-vanishing Euclidean momenta have a well-defined limit as $a \rightarrow 0$. This limit is a power series in the renormalized coupling constant (evaluated at a scale set by the external momenta). The counterterms can be chosen to respect BRST symmetry. Furthermore, their finite parts can be chosen so that the resulting perturbative expansions are equal to those for a theory with 4 degenerate fermions in any desired scheme (e.g. the regularization independent (RI) or $\overline{\mathrm{MS}}$ schemes). This is all a long-winded way of saying that renormalization with lattice regularization works as with other perturbative regulatorsthe additional vertices present on the lattice ( $q \bar{q} g g$ etc.), and the taste violations that are present at large gluon momenta, both lead only to finite renormalizations with a taste-symmetric form.

Renormalizability extends straightforwardly to a theory with $n_{r}$ copies, or "replicas", of unrooted staggered fermions, with $n_{r}$ a positive integer. Counterterms corresponding to divergent fermion loop contributions are simply multiplied by $n_{r}^{\# l o o p s}$, and one obtains renormalized correlators for a theory with $4 n_{r}$ continuum fermions. Thus far the discussion is uncontroversial. The next step is a little less so: I will assume that the "fermion-loop" rule can be applied unambiguously not only to the divergent parts, but also to the finite parts of counterterms needed to bring renormalized correlators into the desired scheme. ${ }^{7}$ This assumption is important, because it implies that, at any order in PT, the counterterms needed to arrive at the desired scheme are polynomials in $n_{r}{ }^{8}$ This claim strikes some (myself included) as very reasonable and others as less obvious. What seems clear, however, is that a demonstration (or refutation) of this supposition would be greatly facilitated by having a proof of the renormalizability of unrooted staggered fermions in hand.

The importance of this discussion is that it allows one to extend the considerations to noninteger $n_{r}$, as first noted in Ref. [50]. The argument goes as follows. Imagine doing a simulation with fermionic weight factor $\operatorname{det}^{n_{r}}\left(D_{\text {stag }}\right)$, which I will generically call the "rooted theory". In perturbation theory, for gluonic correlation functions, this has the effect of multiplying each fermion loop by $n_{r}$ (compared to the unrooted case), irrespective of whether $n_{r}$ is an integer. Such correlation functions can be renormalized simply by substituting the actual value of $n_{r}$ into the counterterms determined for integral $n_{r}$. This follows because both the bare correlators and the counterterms are polynomials in $n_{r}$, and thus so are the renormalized correlators. Since, by assumption, the latter are finite for all positive integer $n_{r}$, the coefficients of the polynomial are finite, and thus finiteness holds for all $n_{r}$. Analytic continuation from the positive integers is possible because we know that the form is polynomial.

This establishes the renormalizability of gluonic correlators in the rooted theory. Note that no additional types of counterterm are needed. However, we need a stronger result, namely that the renormalized correlators are not only finite but are in fact equal to those in the desired scheme with $N_{f}=4 n_{r}$ fermions for any $n_{r}$. This follows from the assumption that the finite parts of the counterterms are also polynomial in $n_{r}$. It also uses the fact that correlators in the desired scheme with $N_{f}$ flavors are, at any finite order in PT, polynomials in $N_{f}$. Since we know that the lattice regularization reproduces these polynomials when $N_{f}=4 n_{r}$ with $n_{r}$ any positive integer, it also

\footnotetext{
${ }^{7}$ I am excluding here hypothetical schemes in which the renormalization conditions have an explicit non-polynomial dependence on $n_{r}$.

${ }^{8}$ To be completely clear, the issue is not whether counterterms can be chosen so that the correlators are in the desired scheme - this is a corollary of the assumed renormalizability — but rather whether the desired counterterms are polynomial in $n_{r}$.
} 
does so for any $n_{r}$.

There is one loose end in this argument—what about fermionic correlators? Fermionic quantities in actual simulations are composed of "unrooted" propagators, $D_{\text {stag }}^{-1}$. Rooting removes the extra degrees of freedom in the sea, but what about in the valence sector? It seems that we are stuck with $4 n_{r}$ valence tastes, with integer $n_{r}$. Does this not lead to violations of unitarity? This is what I call the "valence rooting" issue (which sounds better than the more descriptive "valence unrooting" issue). I discuss it here in the context of PT and then more generally in sec. 10.

To describe the extra valence quarks one is forced into a partially quenched framework. If $n_{r}=1 / 4$, for example, the theory would have a single sea taste but 4 valence tastes. In practice, one simply uses the full staggered propagators for valence lines and multiplies loops by $n_{r}$. The key point is that, by an extension of the previous argument, it is very plausible that valence fermion correlation functions can be renormalized and related to those in a corresponding continuum PQ theory. I claim further that taste will be unbroken in this continuum theory, essentially because this is what happens in the unrooted staggered theory (where the valence fields are, by definition, unrooted). But in a PQ theory in which sea and valence quarks have the same Dirac operator and masses (because taste is unbroken), there is a unitary, physical sub-sector obtained by projecting onto the appropriate number of tastes ( 1 if $n_{r}=1 / 4$ ) for each valence flavor. ${ }^{9}$ This projection is simple in practice because the staggered propagator (discussed in app. A) is taste invariant. It is in this sense (i.e. after projection) that the rooted staggered PT is physical. Note that the projection is only possible for an integer number of tastes.

The upshot is that it is very plausible that, order by order, PT for rooted staggered fermions (with the " $n_{r}=N_{f} / 4$-th" root) reproduces renormalized correlation functions in the continuum theory with $N_{f}$ flavors, as long as one uses either taste projectors or appropriate averages over tastes in valence loops. Thus not only the $\beta$-function (which can be accessed using purely gluonic correlators) but also the anomalous dimensions of the quark mass and external operators will be as in the desired continuum theory (up to the usual dependence on scheme). In other words, the unphysical features of rooting do not appear (or can be removed) in PT, and we find the same fixed point as with uncontroversial fermions. It is important to stress that this holds only when one sends $a \rightarrow 0$. Scaling violations can, and indeed do, contain unphysical effects.

One might be concerned that the argument just given holds for any $n_{r}$, e.g. $n_{r}=\pi / 4$ leading to $N_{f}=\pi$. It is true that such a theory is renormalizable, so that its renormalized Euclidean correlation functions should have a well-defined continuum limit. What is not implied for general $n_{r}$ is that the resulting PT is unitary, i.e. that it corresponds to a physical Minkowski theory. As already noted above, unitarity will hold only for $n_{r}=N_{f} / 4$ with $N_{f}$ a non-negative integer. Otherwise the resulting PT describes a theory which is irreducibly partially quenched. Another way of saying this is that only for $N_{f}$ a non-negative integer does the corresponding continuum theory have an underlying local Lagrangian, from which unitarity can be shown. Note that no claim of unitarity for the rooted theory for $a>0$ is being made here-the logic is that one sends $a \rightarrow 0$ in Euclidean space and finds that the renormalized correlators are those of a familiar perturbative scheme, which, from standard continuum considerations, is known to be physical.

Let me pause and emphasize an important conclusion:

\footnotetext{
${ }^{9}$ Alternatively, as discussed in app. B, one can average over tastes and multiply by an appropriate overall factor.
} 


It is important to complete the proof of renormalizability for unrooted stag-
gered fermions, and to confirm the assumptions needed to extend this result to
$n_{r}=1 / 4$.

In the last year, Giedt has taken a first step towards a proof by generalizing a power-counting theorem of Reisz to staggered fermions [51].

If we accept that rooting gives the correct fixed point in PT are we done? Does this not imply that the continuum limit of the rooted theory is correct? No! It is a very important step, but there are four issues that remain to be resolved. The first two are not specific to rooted theories, and are unlikely to be problematic. I include them for completeness.

1. One must be in the domain of attraction of the desired fixed point. This must be addressed by simulations. One approach is to study the scaling of $\alpha_{s}$ and compare it to the perturbative predictions, as has been done successfully for pure gauge theories and for QCD with $N_{f}=2$ Wilson fermions.

2. Non-perturbative effects at short distances could invalidate the perturbative analysis. Again, numerical tests of scaling can rule out (or in) this possibility.

The remaining two issues are specific to rooted theories.

3. The BGS argument implies that the rooted theory is unphysical for any $a>0$, while the perturbative fixed point is physical. There is no technical inconsistency since the BGS argument relies on non-perturbative long-distance contributions to correlation functions. There is a need, however, to understand how these two results can be made consistent. There are two possibilities, one "good/ugly", one "bad":

Good/ugly: The non-locality vanishes as $a \rightarrow 0$. As already noted in sec. 5, this possibility is consistent with the BGS analysis.

Bad: The PT analysis is not pertinent: the rooted theory does not run to the perturbative fixed point, and remains non-local even at $a=0$. This might happen if the classification of operators into relevant and irrelevant differs from that in a standard RG framework, which is possible since there is no non-perturbative local single-taste construction of the rooted theory. Crudely speaking this amounts to saying that we do not have experience with, or intuition about, theories with non-local actions, so we should be wary of using standard tools. Note that the example from statistical mechanics suggests that standard tools (here PT) can be used, but, of course, this lesson may not apply to the theory at hand.

To study this issue we need an extended RG framework which can deal with rooting. If the "good/ugly" possibility is favored, then we also need to make plausible a mechanism for the vanishing of non-locality in the continuum limit.

4. We must also consider the rooting problem in the valence sector in a non-perturbative context. That is, even if the sea-sector is properly dealt with by rooting, how do we know that 


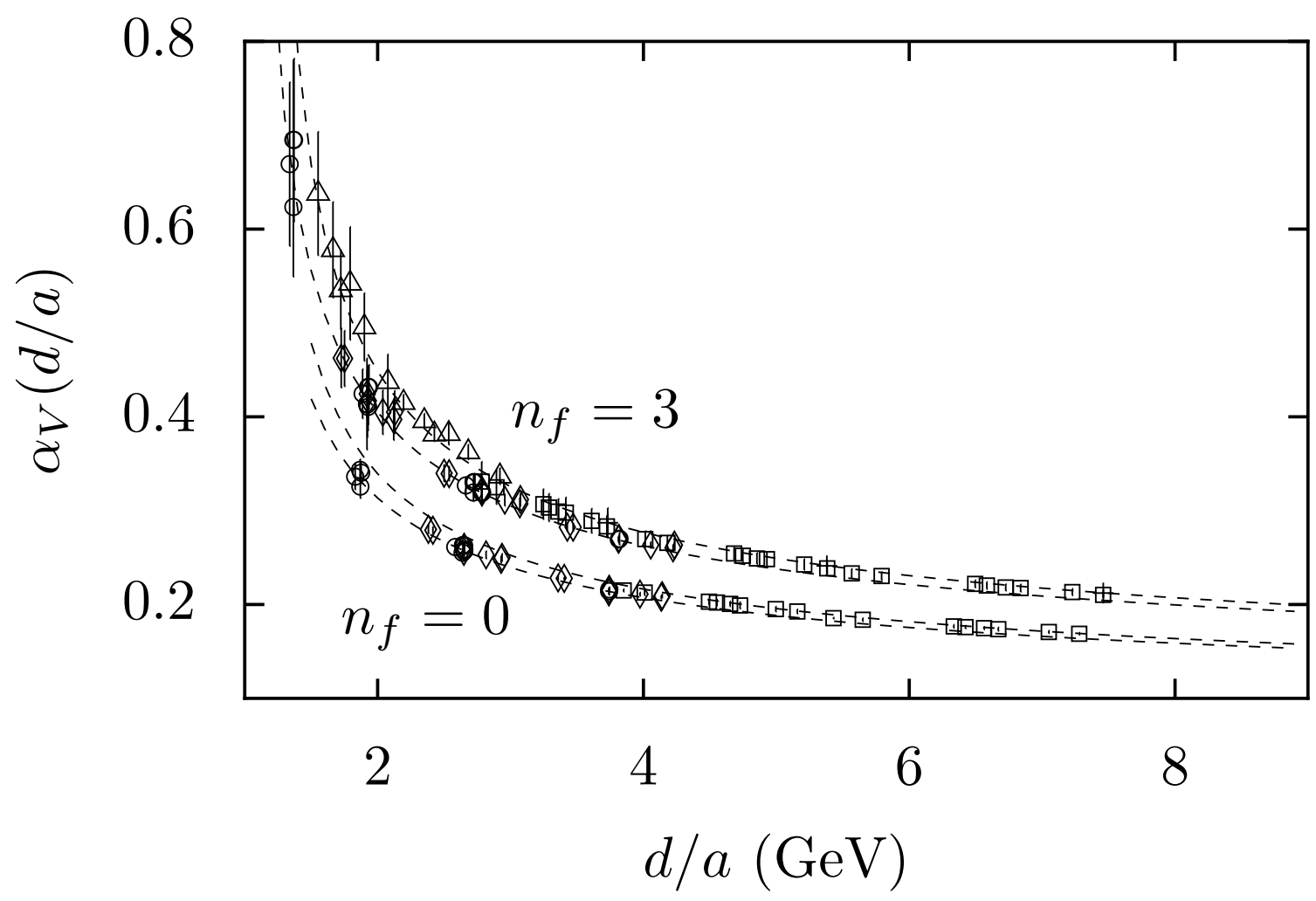

Figure 3: Results for the strong coupling constant, in the potential scheme, versus scale [52].

the valence correlation functions that are calculated in practice give physical results in the continuum limit?

I discuss all of these issues (and more) in the following.

\subsection{Numerical results on scaling}

I begin by briefly showing what is known about the non-perturbative $\beta$-function with rooted staggered quarks. This has been studied in Ref. [52] by calculating Wilson loops numerically and comparing to two-loop (rooted) perturbation theory. This allows an extraction of the coupling constant at a scale which depends on the size of the loop. Note that fermion loops do enter at two-loop order so the perturbative result is sensitive to rooting. The results are shown in Fig. 3 (with " $n_{f}=3$ " referring to the $2+1$ flavor MILC lattices, and " $n_{f}=0$ " to quenched results). The dashed lines show the predicted four-loop scaling for the two theories, with initial values chosen to bracket the simulation results.

What I want to stress here is that the results in the perturbative regime (say $d / a \gtrsim 2 \mathrm{GeV}$ ) are consistent with the predicted scaling. While this is nowhere near as stringent a test as has been made for $N_{f}=2$ Wilson fermions [53], using the Schrödinger functional definition of $\alpha$ [54], it is nevertheless a non-trivial test that QCD with rooted staggered fermions is governed by the desired fixed point. 


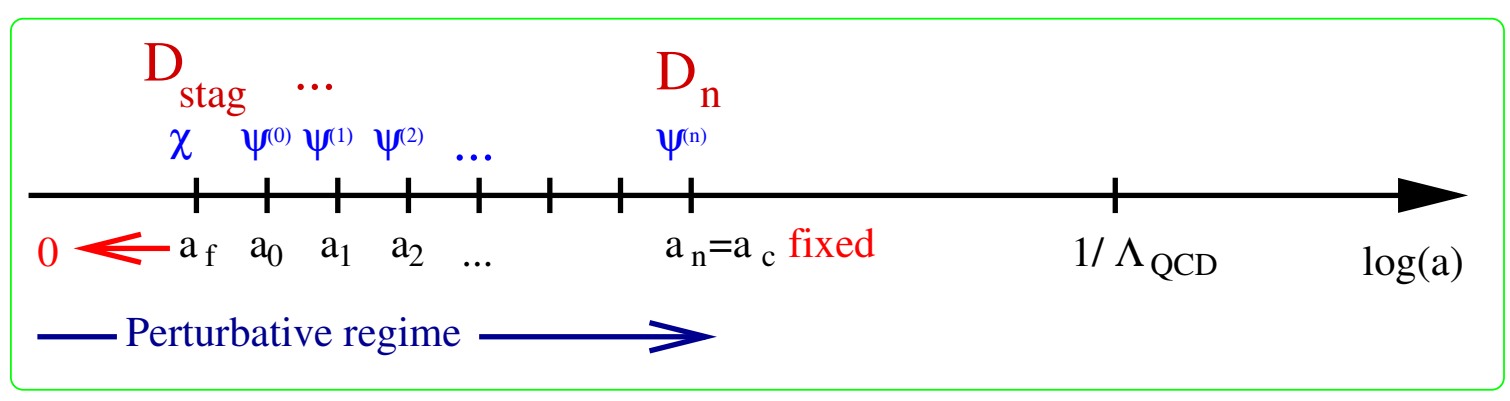

Figure 4: RG framework and notation. Details are discussed in text.

\subsection{Renormalization group analysis}

In this section I describe recent work of Shamir, who studies rooting using an RG framework [16]. The main conclusion is that, given certain assumptions, the non-locality can be bounded and shown to vanish when $a \rightarrow 0$, leaving a theory which is in the correct universality class. (To make this more precise I will need to set up the RG framework.) This is a strong, positive claim for rooted staggered fermions, and, not surprisingly, a key question is the plausibility of the assumptions. In the following I summarize the argument and critically examine the assumptions.

The RG framework is sketched in fig. 4. One starts on the "fine" lattice, with spacing $a_{f}$, and blocks $n+1$ times by a factor of 2 until one reaches the "coarse" lattice, with spacing $a_{c}=2^{n+1} a_{f}$. The aim is to determine the properties of the action on the coarse lattice, whose spacing is held fixed, while $a_{f}$ is sent to 0 by sending $n \rightarrow \infty$. Applying this procedure to, say, Wilson fermions, one would end up with a so-called perfect lattice action at the coarse scale. The issue is, of course, what happens with rooted staggered fermions. Although it does not matter at this stage, it will be assumed later that $a_{c} \Lambda_{\mathrm{QCD}} \ll 1$, so that all the blocking steps are in the perturbative regime.

The fermion blocking is carried out using the "unrooted" fields-we will see later how the results carry over to the rooted theory. One begins on the fine lattice with the one-component staggered field $\chi$, which, in the first step, is transformed to the Dirac-taste basis (labeled $Q$ earlier, but $\psi^{(0)}$ here, following Shamir). This is not a standard blocking transformation, since the number of fermion degrees of freedom is unchanged. The gauge fields are, however, blocked in this first step. Subsequent blocking steps involve standard thinning of both fermion and gauge degrees of freedom, with the fermions remaining in the Dirac-taste basis and labeled $\psi^{(k)}$. Although I must skip over most of the details (such as how gauge and hypercubic invariance are maintained) I do want to mention that fermion blocking is implemented using Gaussian kernels. For example, to move from the $k-1$ 'th to the $k^{\prime}$ th fermionic level, one uses

$$
\int D \bar{\psi}^{(k-1)} D \psi^{(k-1)} \exp \left\{-\alpha_{k}\left[\bar{\psi}^{(k) \dagger}-\bar{\psi}^{(k-1) \dagger} Q^{(k) \dagger}\right]\left[\psi^{(k)}-Q^{(k)} \psi^{(k-1)}\right]\right\},
$$

with $Q^{(k)}$ a local map implementing gauge covariant averaging over a hypercube, and $\alpha_{k}$ a parameter conveniently chosen to be $\sim 1 / a_{k}$.

In a standard RG analysis one would now integrate out all levels of fermions and gauge fields except those on the coarse lattice. This would, however, lead to multifermion interactions (since the $Q^{(k)}$ include gauge fields), and one would lose analytic tractability. Instead, Shamir keeps all levels of the gauge field while integrating out all except the coarse-lattice fermions. All fermionic 
integrals are then Gaussian and can be done explicitly. At each stage one gets a blocked Dirac operator, $D_{k}$, as well as an additional contribution to the blocked gauge action. For present purposes, the key result is

$$
\operatorname{det}\left[D_{\text {stag }}\right] \propto \prod_{k=0}^{n} \operatorname{det}\left[G_{k}^{-1}\right] \operatorname{det}\left[D_{n}\right] \equiv \prod_{k=0}^{n} \exp \left(-4 S_{\text {eff }}^{(k)}\right) \operatorname{det}\left[D_{n}\right]
$$

where $G_{k}^{-1}$ and $D_{n}$ are known explicitly:

$$
G_{k}^{-1}=D_{k-1}+\alpha_{k} Q^{(k) \dagger} Q^{(k)}, \quad D_{k}=\alpha_{k}-\alpha_{k}^{2} Q^{(k)} G_{k} Q^{(k) \dagger}, \quad D_{-1}=D_{\text {stag }} .
$$

The proportionality constant in (8.2) is independent of the configuration.

Thus far this is kinematics: eq. (8.2) is valid configuration by configuration on any ensemble. The important dynamical issue is the locality of the blocked action. Integrating out $\psi^{(k-1)}$ must be shown to lead to an effective gauge action, $S_{\text {eff }}^{(k)}=\operatorname{tr} \ln G_{k} / 4$, and a blocked Dirac operator $D_{k}$, local at scale $a_{k}$. This requires both $G_{k}^{-1}$ and $G_{k}$ to be local at this scale (both appear when taking functional derivatives of $S_{\text {eff }}^{(k)}$ with respect to the gauge field, and $G_{k}$ enters in the expression for $D_{k}$ ). In an inductive argument, $G_{k}^{-1}$ is local because $D_{k-1}$ and $Q^{(k) \dagger} Q^{(k)}$ are, so the real issue is the locality of $G_{k}$. What matters for this are the properties of the eigenvectors of $G_{k}^{-1}$ with small eigenvalues - if there are no small eigenvalues or if their eigenvectors are localized, then $\left(G_{k}^{-1}\right)^{-1}=G_{k}$ will be local. In the free theory there are no small eigenvectors: where the Dirac operator $D_{k-1}$ is small-i.e. for small momenta—the $Q^{(k) \dagger} Q^{(k)}$ term is like a large mass of size $\alpha_{k} \sim 1 / a_{k}{ }^{10}$ This can break down, however, on rough gauge fields, because $D_{k-1}$ need not be small. The situation is similar to that of Wilson fermions with a large negative mass, for which we know that the derivative can become large on rough gauge configurations. Clearly a more sophisticated discussion is needed.

That given by Shamir is drawn from studies of the "mobility edge" of the hermitian WilsonDirac operator with a large negative mass [55]. This operator does have small eigenvalues (indicating that the derivative term is large) but in a suitable region of the phase diagram these are exponentially localized at the scale of the lattice spacing, so that the contribution of the corresponding eigenvectors to the inverse remains local. Only eigenvalues above the mobility edge have delocalized eigenvectors, and as long as this edge remains $\gtrsim 1 / a$, their contribution to the inverse will also be local. Shamir argues that this picture holds also for $G_{k}^{-1}$ (or, more precisely, for the hermitian form $H_{k}=\left(\gamma_{5} \otimes \xi_{5}\right) G_{k}^{-1}$, which is sufficient to establish locality), irrespective of whether the ensemble is rooted or unrooted, as long as one is in the weak coupling regime. Weak coupling enters because numerical results indicate that the mobility edge lies close to the perturbative value, $\sim 1 / a$, in this regime. The irrelevance of rooting is based on the fact that the gauge action dominates the measure for the rough gauge fields.

The outcome is the first key assumption: that $S_{\text {eff }}^{(k)}$ and $D_{k}$ are local at scale $a_{k}$, irrespective of whether the ensemble is rooted or unrooted. I find the argument for this assumption, sketched above, to be plausible. It is "anchored" by the unrooted case: here the theory is local on the fine lattice, and blocking by a local RG transformation should keep it local at each stage, for this is

\footnotetext{
${ }^{10}$ For large momenta, and for $k>0, Q^{(k) \dagger} Q^{(k)}$ is small (it is a projection operator) but this is compensated by the fact that $D_{k-1}$ is large.
} 
a standard RG application. This implies that Shamir's mobility edge argument must work for the unrooted theory.

Accepting the assumption, what have we learned about the rooted theory? The fermion weight factor is then

$$
\operatorname{det}\left[D_{\text {stag }}\right]^{1 / 4} \propto \prod_{k=0}^{n} \operatorname{det}\left[G_{k}^{-1}\right]^{1 / 4} \operatorname{det}\left[D_{n}\right]^{1 / 4}=\prod_{k=0}^{n} \exp \left(-S_{\text {eff }}^{(k)}\right) \operatorname{det}\left[D_{n}\right]^{1 / 4}
$$

The assumption implies that the effective gauge action introduced by blocking, here $S_{\text {eff }}^{(k)}$ (without the factor of 4), remains local on the rooted ensemble. Thus the non-locality in the one-taste theory, which we know is present from BGS, must result from the $\operatorname{det}\left[D_{n}\right]^{1 / 4}$ term. This means that, crudely speaking, the non-locality resides in the IR. This is consistent with my earlier discussion of PT-no unphysical behavior should appear in the perturbative regime- - but goes beyond it, by indicating how non-localities from possible non-perturbative effects at short distances (rough gauge fields) can be controlled.

Now I move to the second part of the argument: bounding the effects of non-locality in $\operatorname{det}\left[D_{n}\right]^{1 / 4}$. This is intertwined with the issue of taste breaking, since it is such breaking that precludes taking the fourth-root of the fermion determinant. It thus makes sense to decompose the blocked Dirac operator as

$$
D_{n}=\underbrace{\widetilde{D}_{n} \otimes \mathbf{1}}_{\text {taste invariant }}+\underbrace{\Delta_{n}}_{\text {taste-breaking }} .
$$

Recall that, given Shamir's first assumption, $D_{n}$ is local on the rooted ensemble, and thus so are the two parts on the r.h.s., as they are obtained by taste-projection. The aim now will be to argue for the second key assumption: $\left|\Delta_{n}\right|$ is bounded from above and vanishes as $a_{f} \rightarrow 0$ (i.e. as $n \rightarrow \infty$ ). This is to be contrasted to $D_{n}$ itself, which is bounded from below by $m\left(a_{c}\right)$, the quark mass at scale $a_{c}$, so that, as long as we work at non-zero $m$, the taste invariant part dominates as $a_{f} \rightarrow 0$. In particular, for small enough $a_{f}$, the rooted determinant

$$
\begin{aligned}
\operatorname{det}\left[D_{n}\right]^{1 / 4} & =\operatorname{det}\left[\widetilde{D}_{n} \otimes \mathbf{1}\right]^{1 / 4} \operatorname{det}\left[1+\Delta_{n}\left(\widetilde{D}_{n} \otimes \mathbf{1}\right)^{-1}\right]^{1 / 4} \\
& =\operatorname{det}\left[\widetilde{D}_{n}\right] \exp \left\{(1 / 4) \operatorname{tr} \ln \left[1+\Delta_{n}\left(\widetilde{D}_{n} \otimes \mathbf{1}\right)^{-1}\right]\right\}
\end{aligned}
$$

can be legitimately expanded in powers of $\Delta_{n}$. The first factor in (8.7) is the determinant for a local one-taste theory with Dirac operator $\widetilde{D}_{n}$, while the second shows explicitly [through the inverses of $\left.\left(\widetilde{D}_{n} \otimes \mathbf{1}\right)\right]$ the non-locality that we knew had to be present. ${ }^{11}$ The key point, however, is that if $\left|\Delta_{n}\right|$ vanishes as $a_{f} \rightarrow 0$ then the non-locality also vanishes in that limit (the second factor becoming unity).

An important property of $D_{n}$ that has been used in this construction is that its taste singlet part, $\left(\widetilde{D}_{n} \otimes \mathbf{1}\right)$, has no doublers $[15,16]$. For this to be true, at least one blocking step must be taken, since it does not hold for the original action, $D_{\text {stag. }}$. This can be seen from eq. (3.3): projecting onto the taste-singlet part reintroduces the doublers.

\footnotetext{
${ }^{11}$ Similarly, one can see, by taking the fourth power of (8.7), how a non-local "gluonic" effective action can lead to taste breaking in the fermion sector of the unrooted staggered theory, as advertised in sec. 5 .
} 
The local theory we obtain as $a_{f} \rightarrow 0$ contains a single taste (per flavor) with action $\sum \bar{q} \widetilde{D}_{n \rightarrow \infty} q$, but has the unusual feature of having all the blocked gauge fields present. Integrating out all except the coarse lattice gauge fields will yield a complicated action involving multifermion terms. This complication is not, however, a theoretical problem. What matters is that the resulting action is local at scale $a_{c}$, and has the same symmetries as, say, lattice QCD with overlap fermions-flavor and the symmetries of the blocked lattices (hypercubic and translation invariance). It is thus in the same universality class as QCD.

I have been rather cavalier with the limit $n \rightarrow \infty$. This is addressed by Shamir by introducing "reweighted" theories in which one replaces $\operatorname{det}\left[D_{n}\right]^{1 / 4}$ with $\operatorname{det}\left[\widetilde{D}_{n}\right]$ for finite $n$. Now one can integrate out a finite number of blocked gauge fields to obtain a complicated but local single-taste action, which is manifestly in the QCD universality class. One can further show, given Shamir's second assumption, that the difference between this reweighted theory and rooted staggered fermions vanishes as $a_{f} \rightarrow 0$. In other words, there is a sequence of local theories labeled by $n$, all in the QCD universality class, which, as $n \rightarrow \infty$, become closer and closer to the rooted staggered theory having the same fine lattice spacing $a_{f}$. Working with these reweighted theories allows one to avoid the possibility, raised at the end of the previous subsection, that non-localities can somehow invalidate the perturbative analysis of RG scaling.

I now return to sketch the argument leading to the assumption that $\left|\Delta_{n}\right|$ is bounded and vanishes as $a_{f} \rightarrow 0$. There are two ways in which taste is broken and correspondingly two parts to the present argument. ${ }^{12}$ The first is that fine lattice gauge fields with momentum components $\sim \pi / a_{f}$ lead to taste violating quark-antiquark-gluon vertices. This is very well known, and an explicit example is given in app. A. What is peculiar about the present RG set-up is that such gluons are not integrated out, and so can lead to taste violations in $D_{n}$, since (tracing back the RG transformations) this depends on the gauge fields on all but the coarse lattice. Such contributions to $\Delta_{n}$ are suppressed, however, by the gluon functional weight. In perturbation theory the gluons are far off-shell, and when they are finally integrated out, lead to taste-violating four-fermion interactions, explicitly suppressed by $1 / k^{2} \propto a_{f}^{2}$ up to logarithms [23, 57, 58, 59].

The second source of taste violation is that present already in the second term in the free theory action (3.3). The lowest dimension operator in this term is $\sum_{\mu} \bar{Q}\left(\gamma_{5} \otimes \xi_{\mu} \xi_{5}\right) \partial_{\mu}^{2} Q$, which has dimension 5 and thus comes with a factor of $a_{f} .{ }^{13}$ Shamir argues that, as the fermions are integrated out, the scaling of the contributions of the covariant form of this operator will have the standard form - the overall factor of $a_{f}$ will remain, up to logarithmic corrections, and $\partial_{\mu}^{2}$ gives $\sim 1 / a_{c}^{2}$. Standard scaling is expected because, from Shamir's first assumption, the interactions are local in the range from $a_{f}$ to $a_{c}$.

The arguments leading to the assumption that $\left|\Delta_{n}\right| \rightarrow 0$ are the least well developed part of

\footnotetext{
${ }^{12}$ The following discussion differs substantially from that in my talk, due to an improved understanding developed in discussions with C. Bernard, M. Golterman and Y. Shamir. A more thorough discussion appears in Refs. [56, 16].

${ }^{13}$ It is probable that taste breaking from this source is actually absent at $O\left(a_{f}\right)$. In the unrooted theory one can argue using the Symanzik improvement program that there are no $O(a)$ corrections with staggered fermions [61, 60]. This implies that there is a field redefinition which removes the $O(a)$ term in eq. (3.3), even in the interacting theory, as discussed by Luo [60]. Since this is an essentially kinematic result, it is plausible that it carries over to the rooted ensemble. Taste violation is then moved to higher order in $a_{f}$. Since I am not making use of the dependence on $a_{f}$, I do not pursue this point further.
} 
Shamir's approach. It is important to check that there are no surprises when one develops perturbation theory for the non-standard RG setup. Nevertheless, even without this check, I think the conclusion is plausible. This is again because of the "anchor" provided by the unrooted theory, together with the plausible claim that "rooting works" in PT (as discussed in sec. 8.1), and the fact that the RG running is in the perturbative domain. For a more thorough discussion see Ref. [56].

In summary, Shamir's analysis provides a RG framework which is able to deal with the rooted determinant despite the lack of a local one-taste theory. Accepting two key assumptions, one can see how the non-locality implied by BGS can be present for non-zero lattice spacing and yet vanish in the continuum limit. This is the "good/ugly" option from the previous sub-section. Although the RG framework is non-standard, it is plausible that the analysis of operators in PT into relevant, marginal and irrelevant still holds, thus making the "bad" option unlikely.

Clearly more work would be welcome, in particular to test the key assumptions. Both can be tested numerically, and the second assumption can also be tested in PT.

\subsection{Understanding non-locality using chiral perturbation theory}

Effective chiral theories turn out to provide an alternative and powerful avenue to tame the nonlocality. In this section I describe the recent work of Bernard [17], who has shown the following result.

If partially quenched staggered chiral perturbation theory (S $\chi$ PT) is a valid
effective field theory (EFT) for unrooted but partially quenched staggered
fermions, and accepting some plausible technical assumptions, then the cor-
rect EFT for rooted staggered fermions is "S $\chi$ PT with the replica trick"
$(\mathrm{rS} \chi \mathrm{PT})$.

Here the EFTs describe the physics of PGBs - their interactions and those with other, heavier, hadrons. Bernard's result is clearly important, and it was a surprise to me that one could derive such a strong statement. I will attempt here to get to the essence of the derivation without going into too much detail.

The implication of the result is that we know the correct EFT describing the long-distance physics of rooted staggered fermions at small, but non-zero, lattice spacings, namely rS $\chi$ PT [59, 62]. I stress that the lattice spacing is an explicit parameter in this EFT, with discretization errors introduced following the method of Ref. [63], using Symanzik's effective Lagrangian [64]. RS $\chi$ PT has two key properties. First, it is unphysical for $a \neq 0$, exhibiting, for example, violations of unitarity. Second, the results go over to those of standard $\chi \mathrm{PT}$ for QCD when $a=0$, as long as one considers the appropriate external states. (The latter point is discussed further in sec. 9.) These are exactly the properties that must hold in order both to be consistent with the BGS argument and obtain the correct continuum limit. What is new here is not that $\mathrm{rS} \chi \mathrm{PT}$ has these properties, but rather that this EFT - with the apparently ad hoc feature of adding factors of $1 / 4$ by hand to represent rooting in the underlying theory [62] — can be shown to be correct.

The importance of this result is twofold. First, it establishes that the continuum limit of the PGB physics in the rooted staggered theory is that of QCD (modulo one caveat about low-energy coefficients [LECs] discussed below). Second, it "tames" the non-locality which is present away from the continuum limit: the unphysical consequences are known explicitly for the properties of 
PGBs and their interactions with each other and with other hadrons. Perhaps "known" is too strong a term: the unphysical features can be parameterized in terms of LECs in the EFT. In any case, the fitting that has been done using $\mathrm{rS} \chi \mathrm{PT}$, which has proven essential in practice, is now on a much firmer theoretical footing.

I have emphasized in my formulation of Bernard's result the need to assume the validity of PQ $\chi$ PT. I have done so both because I think this is the least well established assumption in his analysis, ${ }^{14}$ and because it helps one understand the result. Let me discuss these two points in turn. What is the status of PQ $\chi \mathrm{PT}$ ? PQ theories are unphysical, and the arguments used to justify EFTs in general [65] and $\chi \mathrm{PT}$ in particular [66] only partly apply. One has to make additional assumptions, as discussed in Refs. $[67,68]$. Nevertheless, I do not see any reason at present to doubt the validity of PQ $\chi$ PT: it appears to correctly represent the unphysical nature of the underlying PQ theory, and is supported by a growing body of numerical evidence (an example of which was noted in sec. 6 above). I am partly stressing this point to encourage further study of the issue.

Now I return to the unexpected nature of the result: how is one able to determine the EFT for an underlying theory which is unphysical? The key points are that the rooted theory has a precise formulation as a functional integral, and that, crudely speaking, one is able to trade rooting for partial quenching. While this is superficially just trading one unphysical feature for another, it is actually significant progress. The validity of PQ theories is a general issue, one that can be studied with any fermion discretization and also in the continuum limit, whereas rooting is, in practice, staggered-specific. Furthermore, the underlying PQ theory has a local Lagrangian formulation (albeit involving ghosts) which is not available for the rooted theory.

To explain these comments in more detail I now present my distillation of Bernard's argument. Consider the partition function for four flavors of rooted staggered fermions with non-degenerate masses:

$$
\begin{aligned}
Z^{\mathrm{root}}\left(m_{1}, m_{2}, m_{3}, m_{4}\right) & =\int d \mu_{g}\left\{\prod_{i=1}^{4} \operatorname{det}\left[D_{\mathrm{stag}}\left(m_{i}\right)\right]\right\}^{1 / 4} \\
& =\int d \mu_{g}\left\{\prod_{i=1}^{4} \exp \left[\frac{1}{4} \operatorname{tr} \ln D_{\text {stag }}\left(m_{i}\right)\right]\right\},
\end{aligned}
$$

where $d \mu_{g}$ is the gluonic functional measure (in which resides, implicitly, the strong coupling constant). There are two particularly interesting choices of quark masses. First, setting all the masses equal, one obtains the theory with one unrooted staggered flavor:

$$
Z^{\text {root }}(m, m, m, m)=\int d \mu_{g} \operatorname{det}\left[D_{\text {stag }}(m)\right] \equiv Z^{\text {stag }}(m) .
$$

This is a physical theory which anchors the analysis. Second, one can obtain the "MILC" partition function as follows:

$$
Z^{\mathrm{MILC}}\left(m_{\ell}, m_{s}\right)=Z^{\mathrm{root}}\left(m_{\ell}, m_{\ell}, m_{s}, \sim 1 / a\right) .
$$

Sending one of the quark masses to the lattice cut-off does lead to a renormalization of the coupling constant (and thus to a shift in the lattice spacing), but this is not important for the issue at hand, namely the determination of the form of the EFT.

\footnotetext{
${ }^{14}$ Here I differ from Bernard's presentation, which emphasizes the need for two technical assumptions. These are mentioned below, but I do not have time to discuss them in detail. I find both assumptions very plausible.
} 
Clearly one can move from $Z^{\text {stag }}$ to $Z^{\mathrm{MILC}}$ by varying the quark masses. The strategy of the argument is to do so by calculating all derivatives of $Z^{\text {stag }}$ w.r.t. the $m_{i}$ at the degenerate point, and then summing the series. As shown by Bernard, this can be done within the EFTs. Using this method, one is able to start from a physical theory $\left(Z^{\text {stag }}\right)$ with known EFT and obtain the EFT for $Z^{\mathrm{MILC}}$. The simple words "summing the series" summarize the main technical analysis in Ref. [17], which I will not reproduce. The two technical assumptions needed are (i) that summing the series gives the complete answer-i.e. that there are no non-analyticities, which is plausible since all $m_{i}$ are greater than zero at all stages (so that all particles are massive); and (ii) that there are no unexpected issues in the EFT when one decouples the fourth quark $\left(m_{4} \rightarrow \sim 1 / a\right)$.

The derivatives one needs are illustrated by the following simple example:

$$
C(x, y)=\left.\frac{\partial^{2} \ln Z^{\mathrm{root}}}{\partial m_{4}(x) \partial m_{4}(y)}\right|_{m_{i} \text { equal }}=\left\langle\frac{1}{4}{ }^{2} \bigcirc \bigcirc \mathrm{y}-\frac{1}{4} \mathrm{y}\right\rangle_{Z_{(\mathrm{m})}^{\mathrm{stag}}}
$$

Here the lines indicate propagators - elements of $D_{\text {stag }}^{-1}$. To obtain such results one must treat the quark masses as space-time dependent sources. Inverses of the unrooted $D_{\text {stag }}$ appear because of the form of eq. (8.9), with rooting leading to the factors of $(1 / 4)^{\# \text { loops }}$. Although the expression on the r.h.s. is evaluated in the unrooted ensemble, $Z^{\text {stag }}(m)$, it is not a physical combination of correlators, as it cannot be obtained by taking derivatives w.r.t. the common mass $m$. Such derivatives lead to the wrong relative factors of $1 / 4$ between the two terms. The expression on the r.h.s. is in fact a quantity in a PQ theory with partition function

$$
\begin{aligned}
& Z^{\mathrm{PQstag}}\left(m, M_{V}, \widetilde{M}_{V}\right)=\int d \mu_{g} \operatorname{det}\left[D_{\text {stag }}(m)\right] \times \\
& \prod_{i=1}^{N_{V}} D \bar{\chi}_{V_{i}} D \chi_{V_{i}} D \widetilde{\chi}_{V_{i}}^{\dagger} D \widetilde{\chi}_{V_{i}} \exp [-\underbrace{\bar{\chi}_{V_{i}} D_{\text {stag }}\left(M_{V, i j}\right) \chi_{V_{j}}}_{\text {valence quarks }}-\underbrace{\tilde{\chi}_{V_{i}}^{\dagger} D_{\text {stag }}\left(\widetilde{M}_{V, i j}\right) \widetilde{\chi}_{V_{j}}}_{\text {ghost quarks }}]
\end{aligned}
$$

with the explicit rewriting of $C(x, y)$ being

$$
\left.\frac{\partial^{2} \ln Z^{\text {root }}}{\partial m_{4}(x) \partial m_{4}(y)}\right|_{m_{i}=m}=\left.\frac{1}{4^{2}} \frac{\partial^{2} \ln Z^{\mathrm{PQstag}}}{\partial M_{V, 11}(x) \partial M_{V, 22}(y)}\right|_{M_{V}=\widetilde{M}_{V}=m}+\left.\frac{1}{4} \frac{\partial^{2} \ln Z^{\mathrm{PQ} s t a g}}{\partial M_{V, 12}(x) \partial M_{V, 21}(y)}\right|_{M_{V}=\widetilde{M}_{V}=m} .
$$

This illustrates how rooting can be traded for partial quenching. The need to add factors of $1 / 4$ by hand in the PQ theory betokens the appearance of such factors in rS $\chi$ PT.

Similar identities hold for any number of derivatives. In Bernard's analysis they are applied to the EFTs, i.e. to the partition functions which generate the long-distance parts of correlation functions. By assumption, we know the EFT for $Z^{\mathrm{PQstag}}$ - it is the partially quenched version of the "original" staggered $\chi \mathrm{PT}$ of Lee and myself [59]. By evaluating the derivatives in the PQ theory, and summing the multivariate Taylor series, one can construct the EFT describing the rooted staggered theory. As announced, the result is $\operatorname{rS} \chi \mathrm{PT}[62] .{ }^{15}$

Now I return to the caveat mentioned above. Bernard's argument as given establishes that the EFT has the same form as rS $\chi$ PT, but not that the LECs in it have the same values as those in 3 flavor $\chi$ PT. In other words, it remains possible that the $\chi$ PT obtained when $a \rightarrow 0$, while having

\footnotetext{
${ }^{15}$ In the literature, this is referred to as simply $\mathrm{S} \chi \mathrm{PT}$, without the " $\mathrm{r}$ ", because in practice one only uses staggered chiral perturbation theory in the presence of rooting. Here I keep the " $r$ " for the sake of clarity.
} 
the same form as that for continuum QCD, has different LECs. To show that the LECs are those of QCD $\chi$ PT one must, at present, rely on the RG argument of the previous section to demonstrate that the continuum theory is QCD and not just QCD-like. ${ }^{16}$ It would be clearly better to avoid dependence on the RG argument, and I think this should be possible. The theoretical issue here has nothing to do with rooting. For example, it does not arise in the 4-flavor rooted theory, where Bernard's arguments show that the LECs in the corresponding rS $\chi$ PT are indeed those of the EFT for the QCD-like theory with 4 light flavors. The issue is what happens to the LECs when one decouples the fourth flavor.

In summary, the EFT approach gives both another line of argument showing that the continuum limit of rooted staggered fermions is correct (in the PGB sector) and provides explicit formulae with which to do the chiral and continuum extrapolations. It is complementary to the RG approach, which is general (not restricted to the PGB sector or the chiral regime) but does not provide explicit formulae.

\section{Diseases of rooted staggered fermions?}

The title of this section is adapted from that of a contribution to this conference by Creutz [19]. This is based on a recent paper [18] in which he argues that rooted staggered fermions have fundamental problems related to having too many symmetries. In the terminology of my introduction, Ref. [18] argues that rooted staggered fermions are "bad". It is by now clear, as shown in a rebuttal by Bernard, Golterman, Shamir and myself (BGSS) [20], that the arguments of Ref. [18] do not demonstrate this. What they do highlight, however, is the delicate nature of the continuum limit of the rooted staggered theory, and the need to work at non-zero quark masses while taking this limit. The issue is then whether one should view this situation (which I will explain in the following) as a "disease" or rather an ugly, but tolerable, feature.

The essence of Creutz's concern is that the $D_{\text {stag }}$ residing in the rooted determinant retains the symmetries of the unrooted theory. In particular, it retains the softly broken $U(1)_{\varepsilon}$ symmetry. In the single-component basis this rotates the quark mass $m$ into $m \exp \left[i \varepsilon(n) \theta_{\varepsilon}\right]$, where $\varepsilon(n)= \pm 1$ for even/odd sites. Thus when the rotation angle is $\theta_{\varepsilon}=\pi$ the quark mass changes sign. It follows that $\operatorname{det}\left[D_{\text {stag }}(m)\right]=\operatorname{det}\left[D_{\text {stag }}(-m)\right]$ on all configurations, and thus that the rooted determinant is independent of the sign of $m$,

$$
\operatorname{det}^{N_{f} / 4}\left[D_{\text {stag }}(m)\right]=\operatorname{det}^{N_{f} / 4}\left[D_{\text {stag }}(-m)\right] .
$$

Here I have generalized to a theory which, if rooting is valid, gives $N_{f}$ flavors in the continuum limit, and made use of the fact that the positive root is taken by definition. Equation (9.1) implies that the partition function of the rooted theory is an even function of $m$ for any $N_{f}$. Now consider the theory in a finite (but arbitrarily large) volume and for $a \neq 0$, i.e. before any limits have been taken. Then the fermionic weight is a finite polynomial in $m$ and (since all eigenvalues have non-zero imaginary parts, except possibly on a measure-zero set of configurations) can have no non-analyticities for real $m$. This implies that the partition function depends on $m^{2}$ (rather than the even, but non-analytic, function $|m|$ ). Again this is true for all $N_{f}$.

\footnotetext{
${ }^{16} \mathrm{An}$ alternative approach was discussed here by BGS in which the EFT is obtained more directly using the reweighted local theories mentioned in the previous section [56].
} 
Now we come to the apparent contradiction noted by Creutz. If $N_{f}$ is odd, e.g. $N_{f}=1$, then we know that the continuum theory has no $m \rightarrow-m$ symmetry, because the $U(1)_{A}$ transformation that could change the sign of $m$ is anomalous. Thus the continuum partition function is expected to be a general function of $m$, containing both even and odd powers. To make this less formal we can consider the lattice theory with overlap fermions, for which the same considerations hold for any lattice spacing. Thus we are led to the problem: how can the rooted staggered theory, whose partition function is even in $m$ for any non-zero $a$, have a continuum limit without this symmetry?

The escape from this apparent paradox is fairly simple, and depends on two facts. First, that, by construction, rooting gives a continuum theory with a positive mass, $|m|$, irrespective of the sign of the bare lattice mass. And, second, that non-analyticities can occur since we are taking a limit, $a \rightarrow 0$, in which the number of degrees of freedom goes to infinity. Thus it is perfectly possible for the limit to produce a general function of $|m|$. This can then match the continuum form for positive $m$.

This shows that there is no logical inconsistency with rooted staggered fermions, but we would clearly like more, namely an argument showing how the non-analytic form $|m|$ can appear. BGSS argue as follows. We know that odd powers of $m$ arise in the continuum theory from zero modes of $D_{\text {cont }}$, since these are unpaired. The simplest example is the sector with unit topological charge $(v=1)$, for which, on any configuration, and considering only $N_{f}=1$ for brevity,

$$
\begin{aligned}
\operatorname{det}\left[D_{\text {cont }}(m)\right] & =m F_{\text {cont }}\left(m^{2}\right) \quad(v=1) \\
F_{\text {cont }}\left(m^{2}\right) & =\prod_{\lambda>0}(i \lambda+m)(-i \lambda+m)=\prod_{\lambda>0}\left(\lambda^{2}+m^{2}\right)>0,
\end{aligned}
$$

where the $\lambda$ are real, and the pairing is due to the $U(1)_{A}$ transformation. This formal expression, and those that follow, can be regulated using overlap fermions, for which one can define topological charge, and an index theorem, for $a \neq 0$. The spectrum of $D_{\text {stag }}$ will differ from that of $D_{\text {cont }}$ in two essential ways. First, each eigenvalue of $D_{\text {cont }}$ will be replaced by a quartet, split by an amount which is expected to vanish in the continuum limit. ${ }^{17}$ The pairing of eigenvalues with opposite signs of the imaginary part still holds, now because of the $U(1)_{\varepsilon}$ symmetry. In this way $F_{\text {cont }}\left(m^{2}\right)$ is replaced by $F_{\text {stag }}\left(m^{2}\right)^{1 / 4}$-both even, positive functions of $m$. The second difference between the spectra is that the zero mode of $D_{\text {cont }}$ will become a quartet of non-zero modes, arranged into two pairs because of $U(1)_{\varepsilon}$. There are no exact zero modes because staggered fermions have no index theorem. Thus we expect

$$
\left\{\operatorname{det}\left[D_{\text {stag }}(m)\right]\right\}^{1 / 4}=\left\{\left[\left(\lambda_{1}^{\text {stag }}\right)^{2}+m^{2}\right]\left[\left(\lambda_{2}^{\text {stag }}\right)^{2}+m^{2}\right] F_{\text {stag }}\left(m^{2}\right)\right\}^{1 / 4},
$$

with $\lambda_{1,2}^{\text {stag }} \propto a$. Note that this is a manifestly even function of $m$, as expected from the argument above. If we now take $a \rightarrow 0$, however, we obtain the claimed non-analyticity:

$$
\left\{\left[\left(\lambda_{1}^{\mathrm{stag}}\right)^{2}+m^{2}\right]\left[\left(\lambda_{2}^{\mathrm{stag}}\right)^{2}+m^{2}\right]\right\}^{1 / 4} \underset{a \rightarrow 0}{\longrightarrow}|m| .
$$

This discussion shows how the desired continuum limit can be achieved if, on the dominant gauge configurations, the eigenvalues tend to a particular distribution as $a \rightarrow 0$ (degenerate quartets,

\footnotetext{
${ }^{17}$ Whether this splitting is expected to vanish as $a$ or as $a^{2}$ is not clear. This is discussed briefly below, and in more detail in Ref. [69]. All I need here is that it vanishes, so I use the weaker assumption of $O(a)$ splittings.
} 
with the expected numbers of near-zero modes). Numerical studies of eigenvalues find results consistent with this behavior [70]. While this is an important check of rooted simulations, it will not convince a skeptic, since one does not obtain a physical understanding of the observed behavior. For this one has to rely indirectly on the arguments of the previous section: if the continuum limit is as claimed, then the eigenvalues must behave as outlined above.

It is clear from eq. (9.5) that, as one reduces the quark mass, one must work at correspondingly smaller lattice spacing in order to maintain the correct mass dependence (up to whatever tolerance one is aiming for). This point, stressed long ago by Smit and Vink [71], is brought out more clearly by considering the condensate. If $N_{f}=1$, the condensate is non-vanishing due to the linear contribution to the partition function from the $v=1$ zero mode

$$
\langle\bar{\psi} \psi\rangle_{\mathrm{cont}}=-\frac{1}{Z_{\mathrm{cont}} V} \frac{\partial Z_{\mathrm{cont}}(m)}{\partial m} \underset{m \rightarrow 0}{\longrightarrow} \text { non-zero constant }
$$

This result holds in any volume, $V$, unlike the condensate in $\mathrm{QCD}$, which vanishes in the chiral limit unless one sends $V \rightarrow \infty$ first. This difference arises because the $N_{f}=1$ condensate is not an order parameter, since the $U(1)_{A}$ symmetry is anomalous. The $N_{f}=1$ condensate is also a general function of $m$. By contrast, the condensate with rooted staggered fermions is an odd function of $m$, since it is obtained by differentiating the even quantity $Z_{\text {stag. The contribution from near-zero }}$ modes dominates as $m \rightarrow 0$ and is

$$
\langle\bar{\chi} \chi\rangle_{\text {stag }} \propto \frac{m\left[\left(\lambda_{1}^{\text {stag }}\right)^{2}+\left(\lambda_{2}^{\text {stag }}\right)^{2}+2 m^{2}\right]}{\left\{\left[\left(\lambda_{1}^{\text {stag }}\right)^{2}+m^{2}\right]\left[\left(\lambda_{2}^{\text {stag }}\right)^{2}+m^{2}\right]\right\}^{3 / 4}} .
$$

In this case [unlike in eq. (9.5)] the limits $a \rightarrow 0$ and $m \rightarrow 0$ do not commute. If one sends $a \rightarrow 0$ first one obtains $\operatorname{sign}(m)$, which gives a non-zero constant when subsequently sending $m \rightarrow 0^{+}$. This is the correct order of limits to obtain the physical answer. By contrast, sending $m \rightarrow 0$ first gives zero.

As above, this is a scenario which shows how rooted staggered fermions can have the correct continuum form, but does not itself demonstrate that the resulting condensate is correct. For this one needs other arguments (those of the previous section) or numerical tests. A very nice test has been carried out by Dürr and Hoelbling using the Schwinger model [21]. In this 2-dimensional model analogous issues arise when considering the square-rooted two-taste staggered determinant, for which the putative continuum limit has a single flavor. The rooted results can be compared to those obtained using a single-flavor overlap determinant, allowing a comparison at non-zero lattice spacing. This comparison is shown in Fig. 5. The rooted staggered result tracks the overlap result for positive masses (the difference at larger $m / e$ being understood as a higher order discretization error) until, at $m / e \approx 0.01$, it plummets to zero (as required by antisymmetry and analyticity). This form is consistent with the BGSS explanation, and illustrates the practical implications of the lack of commutativity of $a \rightarrow 0$ and $m \rightarrow 0$ limits. ${ }^{18}$

As already noted, rooted staggered fermions always correspond to a positive quark mass (irrespective of the sign of the mass in $D_{\text {stag }}$ ). One way to obtain a negative quark mass is to add a $\theta F \widetilde{F}$

\footnotetext{
${ }^{18}$ There is no similar issue for $N_{f}=2$, where the staggered determinant is unrooted. The staggered and overlap results show the expected agreement (and oddness in $m$ ). This is analogous to the $N_{f}=4$ case in four dimensions.
} 


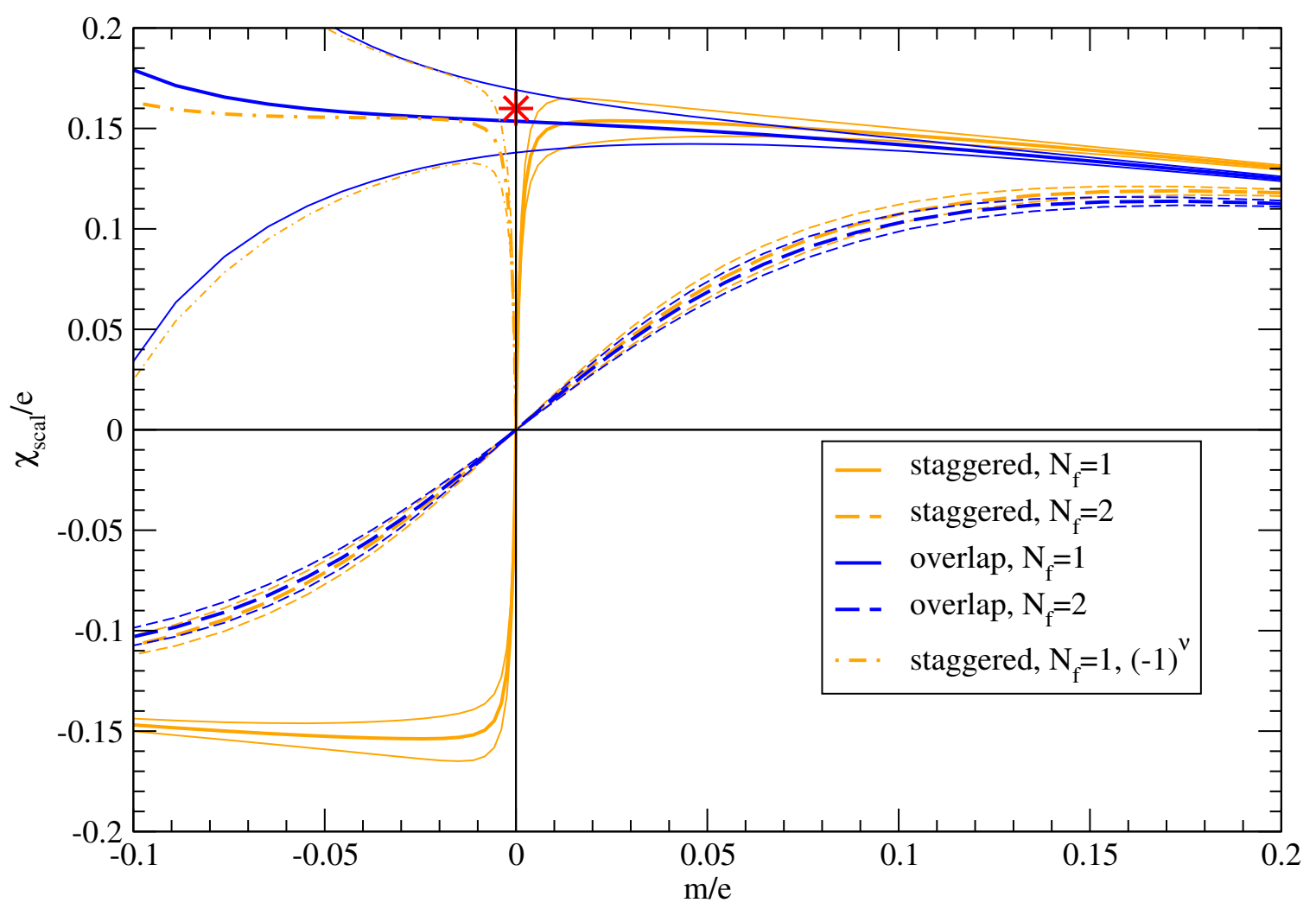

Figure 5: Condensates in the Schwinger model plotted versus quark mass in dimensionless units [21]. Results for $N_{f}=1$ and 2 are shown, using both (rooted) staggered and overlap fermions. Each result shows a central value and a $1-\sigma$ error band. The asterisk shows the known continuum result for the $N_{f}=1$ massless theory. The " $(-1)^{v}$ curve is explained in the text.

term by hand, and set $\theta=\pi$. This is equivalent to adding a weight term $(-1)^{v}$ to the determinant. While this might be numerically challenging for QCD (due to the sign problem) it is tractable for the Schwinger model, as shown by the curved labeled "staggered, $N_{f}=1,(-1)^{v}$ ". This nicely matches onto the overlap results for negative mass (though both have errors which blow up as $|m|$ increases due to the sign problem). Combining this result with that for $m>0$ one reproduces the overlap curve except for a small gap around $m=0$. The BGSS analysis predicts that this gap will shrink to zero width as $a \rightarrow 0$.

How does this analysis impact rooted staggered QCD (with $N_{f}=3$ )? If one works in the isospin symmetric limit, with common mass $m_{\ell}$, and with fixed physical strange quark mass, then the limits $m_{\ell} \rightarrow 0$ and $a \rightarrow 0$ commute for most physical quantities [72]. The issues described above arise when a single quark mass vanishes, e.g. when $m_{u} \rightarrow 0$ with $m_{d}$ and $m_{s}$ fixed and positive. The "staggered, $N_{f}=1$ " curve in Fig. 5 would then qualitatively describe $\langle\bar{u} u\rangle$ as a function of $m_{u}$. The resulting "dip" at $m_{u}=0$ is certainly an unphysical feature of rooted staggered fermions. If one wanted to study QCD in this regime one would have to send $a \rightarrow 0$ before $m_{u} \rightarrow 0$, which would clearly be numerically challenging. Fortunately, this is not a problem in practice for QCD, since it is now almost certain that $m_{u} / m_{d}$ is not close to zero, so one does not come close to the "dip". 19

\footnotetext{
${ }^{19}$ To access the $m_{u}<0$ regime (with its interesting phenomenon of spontaneous CP violation [75]) would require an
} 
The presence of the dip — which is a definite conclusion of Creutz's analysis—provides an interesting challenge. It should be possible to understand its properties using $\mathrm{rS} \chi \mathrm{PT}$ if this is the correct EFT for the rooted theory. The issue is not that $\langle\bar{u} u\rangle$ is odd and thus has a dip-this follows from the same symmetries in $\mathrm{rS} \chi \mathrm{PT}$ as it does at the quark level—but rather that the width of the dip vanishes with $a$. To paraphrase Creutz, how can the UV quantity $a$ enter into what is apparently a long-distance issue involving the IR quantity $1 / m$ ? Let me present a conjecture about how this could happen. This is based on the standard result that $a$ enters into the EFT through the Symanzik quark-level effective Lagrangian [64]. The IR distance scale thus introduced is $1 /\left(a \Lambda_{\mathrm{QCD}}^{2}\right)$. The idea is then that competition between terms in the EFT proportional to $m$ and $a \Lambda_{\mathrm{QCD}}^{2}$ (in fact, it turns out $a^{2} \Lambda_{\mathrm{QCD}}^{3}$ ) can cause the condensate to "swing" from positive to negative values as $m$ is varied. This is exactly what happens for Wilson fermions [73, 63], leading to the Aoki phase, but a similar phenomenon can also occur with staggered fermions [59, 62, 74]. There are many details to be worked out, so this is a speculation, but such an explanation would lead to two general conclusions. First, the width of the dip region would be $\Delta m \sim a^{2}$ implying that the would-be zero-modes have $\lambda_{1,2}^{\text {stag }} \sim a^{2}$ (rather than $\sim a$ ). Second, the explanation would exhibit the non-commutativity of $m \rightarrow 0$ and $a \rightarrow 0$ limits, and thus be consistent with the BGSS analysis. I stress that the lack of commutatitivy is a general phenomenon, not necessarily related to rooting. It occurs, for example, in the flavor-singlet condensate with two-flavors of Wilson fermions (although this is essentially impossible to see in simulations because divergent short-distance contributions swamp those of a non-perturbative origin). Clearly it is important to further develop this potential $\mathrm{rS} \chi \mathrm{PT}$ explanation of the dip.

I now turn briefly to a different, though related, concern raised by Creutz [18]: does the $U(1)_{\varepsilon}$ symmetry lead to Ward identities in the rooted theory which are inconsistent with the proposed continuum limit? More generally, does the extra, unphysical taste symmetry of $D_{\text {stag lead to un- }}$ wanted relations between correlation functions in the rooted staggered theory? The answers are no [20]. While there are additional symmetries and corresponding Ward identities, these have no impact on those correlation functions that become physical in the continuum limit, assuming that this limit is QCD.

The lack of inconsistency can be seen simply from the properties of rS $\chi$ PT. This EFT has an exact $U(1)_{\varepsilon}$ symmetry when $m=0$, and thus has the corresponding exact Ward identities, but nevertheless, when $a \rightarrow 0$, it has a unitary subsector which is $\chi \mathrm{PT}$ for QCD.

Rather than elaborating on $\mathrm{rS} \chi \mathrm{PT}$, however, I think it is clearer to use a formal description of the expected continuum theory with rooted staggered fermions, in which the fermionic weight is 20

$$
\operatorname{det}^{1 / 4}\left[\left(\widetilde{D}_{\text {cont }} \otimes \mathbf{1}\right)+J\right]=\operatorname{det}\left[\widetilde{D}_{\text {cont }}\right] \exp \left\{\frac{1}{4} \operatorname{tr} \ln \left[1+J\left(\widetilde{D}_{\text {cont }} \otimes \mathbf{1}\right)^{-1}\right]\right\}
$$

The idea is that $D_{\text {stag }}$ goes over to the taste symmetric form $\left(\widetilde{D}_{\text {cont }} \otimes \mathbf{1}\right)$. The mesonic sources $J$ allow the construction of correlation functions (in the same way that the quark masses were used

explicit $\theta=\pi$ term added to the action, analogous to that used in Ref. [21].

${ }^{20}$ This simplifies the form that arises from the RG analysis by leaving out the tower of blocked gauge fields. These play no role, however, in the symmetries that are used in the present argument. A less formal discussion can be developed using the reweighted theories, along the lines followed in app. B. 
in the $\chi \mathrm{PT}$ analysis of sec. 8.4). The sources here have general spin, flavor and taste structure. The taste structure means that, although the fermionic weight (when $J=0$ ) is that of the desired one-taste theory, the correlation functions access an extended partially quenched theory involving the extra tastes. Exact Ward identities between these correlators can be derived by doing symmetry transformations on the sources. These include those noted by Creutz, which follow from performing $U(1)_{\varepsilon}$ transformations [corresponding to spin-taste $\left(\gamma_{5} \otimes \xi_{5}\right)$ ], but also many others because of the extended $S U(4)$ taste symmetry. Despite this extended structure, if one uses taste-singlet sources the fermionic weight reduces to that of QCD with physical sources:

$$
J \rightarrow(\widetilde{J} \otimes \mathbf{1}) \quad \Rightarrow \quad \operatorname{det}^{1 / 4}\left[\left(\widetilde{D}_{\text {cont }} \otimes \mathbf{1}\right)+J\right] \rightarrow \operatorname{det}\left[\widetilde{D}_{\text {cont }}+\widetilde{J}\right]
$$

Thus the physical subsector of the extended PQ theory is not constrained by the additional Ward identities. In particular, the precise manner in which the additional Ward identities are saturated is unimportant for the physical subsector. For more details, and explicit examples, see Refs. [20, 56].

What is the upshot of this discussion? Two peculiar properties of rooted staggered fermions have been identified: their behavior when one quark mass vanishes and their need for an extended, PQ continuum limit. Are these diseases (indicating a wrong theory) or ugly features? I think it is now clear that they are ugly features, as long as one accepts as plausible the arguments of sec. 8 that the continuum limit is correct. Let me repeat this logic, lest it get lost in the details: the BGSS arguments show that if the continuum limit is correct, then the peculiar properties are expected. If, however, one looks just at the required behavior of the eigenvalues of $D_{\text {stag }}$, or just at the way in which the "extra" Ward identities must be satisfied (including contributions from ghosts since the theory is PQ), then they may appear implausible. This is not my reaction, but it is that of Creutz [19]. What I want to stress, however, is that this is not the right place to hold the argument over rooting - that belongs in the previous section. The peculiar properties must hold if the continuum limit is correct, and cannot be used to prove the rooting trick wrong by contradiction.

\section{Is there a "valence rooting" problem?}

In this section I discuss what I have called above the "valence rooting" issue. The basic question is how to account for the extra tastes present in valence propagators, although I will pose it in a sharper form shortly. This is an important issue since extensive calculations with valence staggered fermions are being done (leading to several of the "gold-plated" results). It is different from the issue which has been my focus up to now, namely the effects of using a rooted determinant, although the two are closely related. In particular, any resolution of the valence rooting issue certainly requires that the rooting prescription for the determinant leads to the correct continuum limit, i.e. that the premises of the arguments of sec. 8 are correct.

Let me state the issue in a more pointed way, quoting a hypothetical skeptic:

Let me accept that the rooted staggered determinant can be recast as a single-taste local theory in the continuum limit, with Dirac operator $\widetilde{D}_{\text {cont. Does this not mean that }}$ the actual calculations are then being done in a mixed-action theory, since the valence Dirac operator $D_{\text {stag }}$ differs from $\widetilde{D}_{\text {cont }}$ ? Furthermore, unlike in a standard mixedaction set-up we do not know the explicit form of $\widetilde{D}_{\text {cont, }}$ and so we cannot calculate 
sea-quark hadron propagators in order to match valence and sea quark masses, even in the continuum limit. Does this not imply that calculations in the continuum limit will be partially quenched with uncontrolled unitary violations, since we do not know $m_{\text {valence }}-m_{\text {sea }}$ ?

The answer to both these question is no. The essential reason is that applying the same blocking to the valence propagators as to the $D_{\text {stag }}$ in the determinant leads to the same form $\left(\widetilde{D}_{\text {cont }} \otimes \mathbf{1}\right)$. [I am using here $D_{\text {cont }}$ as a shorthand for the $\widetilde{D}_{n \rightarrow \infty}$ used above.] Since the same operator appears in the valence and sea sectors, one obtains $m_{\text {valence }}=m_{\text {sea }}$ without any tuning. Thus unitarity violations associated with $m_{\text {valence }} \neq m_{\text {sea }}$ will be absent. It is true, however, that there are additional valence tastes (because of the " $\otimes \mathbf{1}$ ") and so the continuum theory is partially quenched in this sense. But, as already discussed in the previous section, because $m_{\text {valence }}=m_{\text {sea }}$ this PQ theory has a unitary, physical subsector (that coupled to taste-singlet mesonic sources) for which any potential concerns about partial quenching are irrelevant.

This summary, while accurate, glosses over some important details. Since, as far as I know, there is no thorough discussion of these details in the literature, I present one in appendix B.

The valence rooting issue has also been resolved in one limited, though important, context, that of the chiral EFT describing the long-distance physics of the rooted theory [17]. If the valence and sea-quark Dirac operators differed one would perforce be using a mixed action, so the resulting EFT should be of the mixed-action type [76]. In particular, there would be differences between the masses of charged PGBs composed of the same flavors of valence and sea quarks. But this is not the case. As discussed in sec. 8.4, we know the EFT for rooted staggered fermions to be rS $\chi$ PT, and this does not have the mixed-action form [17]. This is not to say that there are no unphysical effects for $a \neq 0$, but rather that these effects are not of the form resulting from the use of a mixed action.

\section{Rooting at finite temperature and density}

Rooted staggered fermions have been used extensively in simulations of the properties of QCD at non-zero temperature $(T)$ and density $(\mu)$. Studies at $T>0$ (but $\mu=0$ ) are in a mature phase, similar to that of the $T=0$ calculations discussed in sec. 6 above. They are reviewed here by Heller [77] and Stephanov [80], Studies at $\mu \neq 0$ are limited by the sign problem and are still exploratory. They are discussed here in review talks by Schmidt [78], Splittorff [79], and Stephanov [80]. The question I want to address briefly here is whether, compared to $T=\mu=0$, additional issues arise when using rooted staggered fermions at non-zero $T$ and/or $\mu$.

For $T>0, \mu=0$, the answer is no. This is because the PT and RG analyses discussed in secs. 8.1 and 8.3, respectively, are done at short distances, and are unaffected by the boundary conditions on fields necessary to implement $T>0$. What could be a problem in practice, however, is a combination of two factors: first, that the lattice spacings used at $T>0$ are perforce larger than those used at $T=0$, implying larger scaling violations, and, second, that one does not have a S $\chi \mathrm{PT}$ form to fit with in general. The chiral EFT can only be used for the relatively uninteresting regime $T \ll \Lambda_{\mathrm{QCD}} \approx T_{c}$. These factors make it even more important to use improved actions than at $T=0$.

The situation is quite different for $\mu>0$. It has recently been argued by Golterman, Shamir and Svetitsky (GSS) that rooted staggered fermions then face a new problem [22]. A nice summary 
of their argument is given in their presentation here [69]. I give only a brief sketch of this argument and then comment on its implications.

The essential point concerns the distribution of eigenvalues of $D_{\text {stag. }}$ For $\mu=0$, as already noted in sec. 9, the eigenvalues lie on a line of the form $m+i \lambda$, and so do not approach closer than $m$ to the origin. As $\mu$ increases, however, the eigenvalues spread off this line into the complex plane. Once $\mu$ exceeds a critical value the distribution extends into the region with negative real part, and thus overlaps the origin. This critical value is $\mu \approx m_{\pi} / 2$ at $T=0$, but it likely increases with $T$, although how is presently unclear.

Once eigenvalues overlap the origin the definition of rooting becomes ambiguous. In particular, the phase of the determinant becomes ambiguous, which is problematic because the phase plays an essential role in $\mu>0$ physics. To understand this ambiguity, recall that the eigenvalues are expected to lie in quartets, separated by of $O(a)$ [or possibly of $O\left(a^{2}\right)$ for some eigenvalues, as discussed in sec. 9 and in Ref. [69]]. (The quartet structure is not expected to hold if $|\lambda| \sim 1 / a$, but such eigenvalues do not contribute to the uncertainty in the phase of the determinant.) If a quartet lies away from the origin, any value lying "within" the quartet, such as the average $\bar{\lambda}$, provides a sensible definition of its contribution to the rooted determinant. All such choices have similar phases. If, however, the quartet straddles the origin then the phase is ambiguous - the region within the quartet has, by definition, all possible values of the phase.

This ambiguity introduces a systematic error proportional to the fraction of configurations which have one or more eigenvalue quartets straddling the origin. GSS estimate this error to be $\sim a^{3} V^{3 / 2} \Lambda_{\mathrm{QCD}}^{9}$, where $V$ is the space-time volume. ${ }^{21}$ The volume appears because the density of eigenvalues increases with the volume. This result implies that one must take the continuum limit before taking the infinite volume limit - another rather ugly feature of rooted staggered fermions.

How does this impact present calculations at $\mu>0$ ? The simulations which are most clearly effected are those working directly at $\operatorname{Re}(\mu) \neq 0$. The rough estimates of GSS indicate that the problem may be severe for present simulations, but this is controversial, and needs further study. In particular, one needs to look at the distribution of eigenvalues on the configurations produced in the actual simulations - do they lie in quartets, and how frequently do quartets straddle the origin? The impact on the method using analytic continuation from imaginary $\mu$ is less clear. The bottom line is that this is a new issue which must be added to the travails of finite density studies if one uses rooted staggered fermions.

\section{Summary}

In this talk I have tried to provide a rather comprehensive discussion of the status of rooted staggered fermions, concentrating on the core theoretical issues. There has been a lot of progress in the last year, most of it "positive" for rooting, the main exception being the new problems that arise for non-zero chemical potential.

Where do things stand? Are rooted staggered fermions good, bad or ugly? It is clear that we can rule out "good" - the non-locality for $a \neq 0$ ensures a complicated continuum limit. The choice

\footnotetext{
${ }^{21}$ This form assumes the splitting in eigenvalue quartets to be of $O(a)$. The error is parametrically smaller if the splitting is of $O\left(a^{2}\right)$. This does not, however, effect the qualitative conclusions drawn in this section.
} 
is between "bad" (or "diseased") and "ugly". In my opinion, the arguments presented in sec. 8 (using perturbation theory, the renormalization group and $\chi \mathrm{PT}$ ) make plausible that the correct choice is ...ugly. Let me repeat what this means-rooted staggered fermions are unphysical for $a \neq 0$, yet the results go over to those of a physical, single-taste theory in the continuum limit. In practice this very likely requires fitting to the complicated, and one might say ugly, forms of rS $\chi$ PT. In this way the unphysical effects are included in the fit, and in particular in the error estimate.

I have also discussed the important, but secondary, issue of the impact of treating sea and valence quarks differently — crudely speaking, of "rooting one but not the other." I have argued that there is, in fact, no negative impact, as long as one chooses valence correlators appropriately and does the appropriate perturbative matching.

If rooted staggered fermions are indeed "ugly", then the price one pays for rooting is the need for significantly more elaborate fitting, and the concomitant increase in systematic errors. In addition, to obtain the fitting forms one must carry out the $\operatorname{rS} \chi \mathrm{PT}$ calculation anew for each quantity, and this calculation can become very involved, especially those involving baryons or complicated operators. Nevertheless, I stress that, for the mesonic quantities that have been the main focus to date, these complications have been successfully handled, and the resulting errors are small.

My conclusion of "ugly" rather than "bad" clearly rests on an assessment of the assumptions needed to make the RG argument of Shamir, and the $\chi \mathrm{PT}$ argument of Bernard. To me they are plausible, and in some cases very plausible. Plausibility is, however, in the eye of the beholder. Others might find the assumptions implausible. It is thus important to test these assumptions and the good news is that, to varying extents, this is possible. The highest priority items are these:

- Test the two key assumptions of the RG argument of sec. 8.3:

- The required locality of the blocked Dirac operators and the effective gauge actions $S_{\text {eff }}^{(k)}$ can be tested using numerical simulations;

- The required scaling of the taste non-invariant parts of the blocked Dirac operators can be tested using perturbation theory and numerical simulations.

- Strengthen the foundations of partially quenched $\chi \mathrm{PT}$, which is needed to make the argument of sec. 8.4. This is a theoretical issue, but is also tested numerically by PQ simulations with any type of fermion.

- Complete the proof of the renormalizability of unrooted staggered fermions, upon which the perturbative claims for rooted staggered fermions are based.

Also welcome are further numerical tests of rooting, in particular the studies of eigenvalues, and further tests of the validity of rS $\chi$ PT formulae.

Although I have argued that attempts to show the inconsistency of rooted staggered fermions have failed, these arguments have clarified the limitations of rooting. Rooted simulations of QCD are, in practice, restricted to having all quark masses positive and none close to vanishing. The good news is that this allowed regime includes physical QCD. The bad news is that rooted studies of theories other than physical QCD, e.g. one flavor QCD, which are of interest for a number of reasons [81], will be hampered. 
The dispute over rooting has not been, and will likely never be, completely resolved. This is the nature of working in a non-perturbative regime. The best that one could hope for would be to show that rooted staggered fermions have the same continuum limit as that of one of the uncontroversial fermions, i.e. that they are on the same footing as other fermions. While this has not been demonstrated, what I find encouraging is that, with the RG argument, we have a theoretical framework in which we can see both how the required unphysical effects are present and how they vanish in the continuum limit. In particular, the naive statement that taste symmetry is restored in the continuum limit, crudely speaking that $D_{\text {stag }}$ goes over to $\left(\widetilde{D}_{\text {cont }} \otimes 1\right)$, so that the determinant can be fourth-rooted, can now be backed up with a detailed description of the corrections.

How should a non-lattice physicist react? The bottom line I think is that there is substantially more evidence for the validity of rooting now than a year ago. Irrespective of the rooting debate, however, any result obtained with one type of fermions alone would need to be cross-checked with other methods. So perhaps the best news in the last few years is the major strides that have been made in simulating with other fermions. The desired checks will come sooner rather than later.

\section{Acknowledgments}

I am very grateful to Claude Bernard, Philippe de Forcrand, Maarten Golterman, Karl Jansen, Tony Kennedy and Yigal Shamir for many helpful discussions and comments on the manuscript. This research was supported in part by U.S. Department of Energy Grant No. DE-FG02-96ER40956.

\section{A. Perturbation theory with staggered fermions}

In this brief appendix I describe some important features of perturbation theory for staggered fermions that are mentioned in the text.

Perturbation theory for (unrooted) staggered fermions was developed in Ref. [82], and subsequently refined by many authors. Because of doubling, the fermion propagator has $2^{4}$ poles in the Brillouin zone. The positions of these poles are conveniently labeled (in lattice units) as $p_{\mu}=A_{\mu} \pi$, where $A_{\mu}$ is a hypercube vector (all components 0 or 1 ). Each pole is associated with a reduced Brillouin zone, half as wide in all directions $\left(-\pi / 2<p_{\mu}^{\prime} \leq \pi / 2\right)$. A general momentum can then be packaged as $p=p^{\prime}+A \pi$, in which $p^{\prime}$ turns out to be the physical momentum and $A$ the spintaste label. This construction is similar to the position space decomposition described in sec. 3 , but differs because the phase associated with a physical momentum here varies within a spatial hypercube, while that in the position-space construction does not.

An attractive feature of this "momentum-space basis", as it is called for obvious reasons, is that the propagator is taste symmetric:

$$
G^{-1}\left(q^{\prime}+B \pi, p^{\prime}+A \pi\right)=\bar{\delta}\left(q^{\prime}+p^{\prime}\right)\left[\sum_{\mu} i \sin q_{\mu}^{\prime}{\overline{\overline{\left(\gamma_{\mu} \otimes \mathbf{1}\right)}}}_{B A}+m \overline{\overline{(\mathbf{1} \otimes \mathbf{1})}}_{B A}\right] .
$$

Here $\bar{\delta}$ is the periodic $\delta$-function (with period $2 \pi$ ), and the "double-barred" spin-taste matrices are unitarily equivalent to the matrices $\left(\gamma_{B} \otimes \xi_{C}\right)$ appearing in the position-space basis, eq. (3.3). This packaging was introduced by Ref. [26] and refined in Refs. [83, 84]. The result (A.1) is to be 
contrasted to the position-space basis action of eq. (3.3), which has the $O(a)$ taste-breaking term. There is no contradiction between these two results. In particular, the $O(a)$ term in eq. (3.3) can be removed by a field definition and thus does not contribute to on-shell quantities [60]. Nevertheless, the difference between the results in the two bases illustrates why a study of taste-breaking using PT is most straightforward in the momentum-space basis.

Taste $i s$ broken by vertices, and I want to show a simple example since this is referred to in sec. 8.1. This is the $\bar{q} q g$ vertex, which takes the form [85]

$$
V_{\mu}(\underbrace{q^{\prime}+\pi B}_{q}, \underbrace{p^{\prime}+\pi A}_{\bar{q}}, \underbrace{k^{\prime}+\pi C}_{g})=-i g \bar{\delta}\left(p^{\prime}+q^{\prime}+k^{\prime}\right) \cos \left(q_{\mu}^{\prime}+k_{\mu}^{\prime} / 2\right) \overline{\overline{\left(\gamma_{\mu \widetilde{C}} \otimes \xi_{\widetilde{C}}\right)}}{ }_{A B},
$$

assuming that $C_{\mu}=0$ ( $\mu$ being the gluon polarization direction). The new hypercube vector is $\widetilde{C}_{v}=2 \sum_{\rho \neq \nu} C_{\rho}$, the important property of which is that $\widetilde{C}=0$ iff $C=0$. All primed momenta (including that of the gluon) lie in the reduced Brillouin zone (although the result actually holds independent of this).

Taste is broken by the taste matrix $\xi_{\widetilde{C}}$, but only if $C \neq 0$. This in turn implies that the gluon has a large, unphysical momenta ( $\sim \pi / a$ in physical units), and its contribution is suppressed by the gluon action. Gluons with near-physical momenta have $C=0$ and conserve taste.

\section{B. Resolution of valence rooting issue}

In this appendix I sketch how the "valence rooting issue", which has been stated in sec. 10, can be resolved. The argument makes essential use of Shamir's RG analysis [16], and also draws on work done long ago when using staggered fermions to calculate electroweak matrix elements (see, e.g., Refs. [86]). See also the related discussion in Ref. [36]. I assume that rooted staggered fermions lead to a single-taste, physical continuum limit, and in particular that Shamir's RG analysis (discussed in sec. 8.3) is correct. In this sense the valence rooting issue is secondary.

As noted in sec. 10, the issue is that the valence propagators used in practice, $D_{\text {stag }}^{-1}$, are not obviously related to the single-taste operator which resides in the determinant in the continuum limit, $\widetilde{D}_{n \rightarrow \infty}$. The argument of this appendix attempts to establish this relation. There are two parts to the argument: first, dealing with the additional tastes that are present in $D_{\text {stag }}^{-1}$, and, second, showing how blocking relates the valence Dirac operators in essentially the same way as those appearing in the determinant.

Recall from sec. 8.3 that the blocked form of $D_{\text {stag }}$ can be written as a taste invariant piece plus a remainder which vanishes with $a_{f}: D_{n}=\left(\widetilde{D}_{n} \otimes \mathbf{1}\right)+O\left(a_{f}\right)$. The remainder is bounded, allowing the rooted determinant to be expanded as $\operatorname{det}\left(D_{n}\right)^{1 / 4}=\operatorname{det}\left(\widetilde{D}_{n}\right)\left[1+O\left(a_{f}\right)\right]$. The propagator can be expanded similarly, ${ }^{22}$

$$
D_{n}^{-1}(x, y)=\left(\widetilde{D}_{n} \otimes \mathbf{1}\right)^{-1}(x, y)\left[1+O\left(a_{f}\right)\right],
$$

with the taste non-invariant correction vanishing as $a_{f} \rightarrow 0$ for fixed physical quark mass and fixed physical distance $x-y$. In the following, I take $n$ large enough (i.e. take $a_{f}$ small enough relative

\footnotetext{
${ }^{22}$ Strictly speaking, this holds only in the ensemble of configurations produced with the gluonic and fermionic weights. In particular, as noted in sec. 8.3 and app. A, the damping provided by the gluon action is necessary to obtain the smallness of the correction term.
} 
to the fixed coarse lattice spacing $\left.a_{c}\right)$ that the $O\left(a_{f}\right)$ corrections are negligible. It is convenient to make use of Shamir's reweighted theories, in which one changes the fermionic weight from $\operatorname{det}\left(D_{n}\right)^{1 / 4}$ to $\operatorname{det}\left(\widetilde{D}_{n}\right)$. I extend this by using propagators $\left(\widetilde{D}_{n} \otimes \mathbf{1}\right)^{-1}$ in place of $D_{n}^{-1}$. This allows one to work with an exactly taste-invariant theory at finite $n$, and yet obtain results differing only by corrections of $O\left(a_{f}\right)$ from those in the original rooted theory with propagators $D_{n}^{-1}$.

The reweighted theory with propagators $\left(\widetilde{D}_{n} \otimes \mathbf{1}\right)^{-1}$ has the correct number of sea quarks and thus differs from conventional fermions only by the presence of extra tastes in the propagators. This does mean, however, that it is partially quenched. The "fermionic" part of the action can be written $^{23}$

$$
\mathscr{L}_{F}=\sum_{i=1}^{4}\left\{\widetilde{\bar{\psi}}_{i} \widetilde{D}_{n} \widetilde{\psi}_{i}+\bar{\eta}_{i} \widetilde{\psi}_{i}+\widetilde{\bar{\psi}}_{i} \eta_{i}\right\}+\sum_{j=1}^{3} \phi_{j}^{\dagger} \widetilde{D}_{n} \phi_{j},
$$

where the $\widetilde{\psi}_{i}$ are four single-taste fermions, while the $\phi_{j}$ are ghosts (commuting spin-1/2 fields) corresponding to three of these fermions. Having one more fermion than ghost leads to the desired determinant. The sources $\eta$ and $\bar{\eta}$ allow propagators to be generated in the standard way, and since all four tastes are "sourced" the general propagator is the full $\left(\widetilde{D}_{n} \otimes \mathbf{1}\right)^{-1}$. I stress that the PQ set-up is needed because $D_{\text {stag }}^{-1}$ is related to this four-taste propagator, and not the single-taste $\widetilde{D}_{n}^{-1}$.

It should also be kept in mind that all levels of gauge field (from fine to coarse) are still present. The theory can be made to look more conventional by integrating out all but the coarse lattice fields. This gives rise to a multitude of multifermion interactions, but, as noted in sec. 8.3, does not change the universality class. It also does not effect the source terms in the action (B.2), which can still be used in the standard way to generate correlation functions. Nothing essential is gained by integrating out the gauge fields, however, and one looses the simplicity of the fermion action, so I choose not to do this integration in the following.

I can now address the issue of the extra valence tastes. How can one calculate the single-taste quantities of interest? One approach is straightforward: simply construct correlators using sources only of a single taste, say $\eta_{4}$ and $\bar{\eta}_{4}$. In this way, one can calculate any correlation function of interest (meson, baryon, matrix elements, ...) exactly as for uncontroversial fermions. This is possible because taste is unbroken in the reweighted theory. In effect, the procedure replaces $\left(\widetilde{D}_{n} \otimes \mathbf{1}\right)^{-1}$ with $\widetilde{D}_{n}^{-1}$. For example, the charged pion correlator would simply be

$$
C_{\pi}^{1-\text { taste }}(x, y)=\left\langle\operatorname{Tr}\left[\gamma_{5} \widetilde{D}_{n}^{-1}(x, y) \gamma_{5} \widetilde{D}_{n}^{-1}(y, x)\right]\right\rangle_{n^{\prime} \text { th reweighted }},
$$

where quark masses and color indices are implicit, and $x$ and $y$ are points on the coarse lattice. Note that the determinants arising from integrating out the other three tastes of fermions and ghosts cancel, so one is working in a physical subsector of the partially quenched theory.

In practice, however, the calculations that are done do not correspond to using the singletaste approach in the reweighted theory. Instead, all tastes are used, but in such a way that the resulting correlator equals (identically) that of the single-taste construction. This equality can be checked by hand on a case-by-case basis-it involves a purely group-theoretical exercise (really

\footnotetext{
${ }^{23}$ This formulation is similar to, but more general than, that used in sec. 9-see eq. (9.8). Here the sources couple to quark fields rather than to mesons, allowing one to create the more general correlators of the type used to calculate matrix elements and baryons.
} 
just counting) since the dynamics is taste-invariant. ${ }^{24}$ The flexibility of using all tastes allows one to project external operators onto representations of the staggered fermion symmetry group, thus disentangling different contributions to correlators. For example, for mesonic quantities it is often advantageous to use taste $\xi_{5}$, which couples to the lattice Goldstone pion. In the example of the charged pion propagator one then has

$$
C_{\pi}^{1-\text { taste }}(x, y)=\frac{1}{4}\left\langle\operatorname{Tr}\left[\left(\gamma_{5} \otimes \xi_{5}\right)\left(\widetilde{D}_{n} \otimes \mathbf{1}\right)^{-1}(x, y)\left(\gamma_{5} \otimes \xi_{5}\right)\left(\widetilde{D}_{n} \otimes \mathbf{1}\right)^{-1}(y, x)\right]\right\rangle_{n^{\prime} \text { th reweighted }}
$$

The factor of $1 / 4$ cancels the sum over tastes. This approach of averaging over tastes has long been used, e.g. in setting up the calculation weak matrix elements with staggered fermions [86]. They are the analog of the factors of $1 / 4$ introduced in sea-quark loops by rooting.

It should be stressed that the use of reweighted theories allows the flexibility of using different taste projections to be put on a firm footing. Previously (and I am guilty of this) one worked out the counting factors assuming that taste was restored, not taking into account the fact that taste is actually broken for $a \neq 0$.

The discussion so far has shown how one can deal with the extra valence tastes. The end result is that the desired correlators can be built out of propagators $\left(\widetilde{D}_{n} \otimes \mathbf{1}\right)^{-1}$, with appropriate taste projections and normalization factors added by hand. One then uses the result (B.1) above to replace these propagators with $D_{n}^{-1}$, and similarly replaces the reweighted determinant by the original rooted determinant, knowing that one makes only an error of $O\left(a_{f}\right)$ when doing so. Thus, for the example of the charged pion correlator one has

$$
\begin{aligned}
C_{\pi}^{1-\text { taste }}(x, y) & =C_{\pi}^{\text {stag }}(x, y)+O\left(a_{f}\right), \\
C_{\pi}^{\text {stag }}(x, y) & =\frac{1}{4}\left\langle\operatorname{Tr}\left[\left(\gamma_{5} \otimes \xi_{5}\right) D_{n}^{-1}(x, y)\left(\gamma_{5} \otimes \xi_{5}\right) D_{n}^{-1}(y, x)\right]\right\rangle_{\text {coarse }} .
\end{aligned}
$$

The expectation value (labeled "coarse") involves an integration over all levels of gauge field, weighted by the original gauge action and blocking kernels and by $\operatorname{det}\left(D_{n}\right)^{1 / 4}$. Just to be completely clear, the message of eq. (B.6) is that the (theoretically uncontroversial) correlator we want to calculate, $C_{\pi}^{1-\text { taste }}$, is related (up to a controlled error) to a rooted staggered correlator, $C_{\pi}^{\text {stag }}$.

Now I can return to the second issue, namely that we actually calculate using $D_{\text {stag }}^{-1}$ rather than $D_{n}^{-1}$, so that $C_{\pi}^{\text {stag }}$ is not yet of the form we actually calculate. The key point is that, by "undoing" the fermionic blocking, one can rewrite the blocked propagator in terms of the original fine-lattice propagator. This is done by repeated use of the result

$$
D_{k}^{-1}=\alpha_{k}^{-1}+Q^{(k)} D_{k-1}^{-1} Q^{(k) \dagger}
$$

and leads to the schematic form

$$
D_{n}^{-1}(x, y)=c \delta_{x, y}+\sum_{x^{\prime}, y^{\prime}} \mathscr{Q}\left(x, x^{\prime}\right) D_{\text {stag }}^{-1}\left(x^{\prime}, y^{\prime}\right) \mathscr{Q}^{\dagger}\left(y^{\prime}, y\right)
$$

Here $x$ and $y$ are coarse lattice points, while $x^{\prime}$ and $y^{\prime}$ lie on the fine lattice. The constant $c$ can be expressed in terms of the $\alpha_{j}$ 's. $\mathscr{Q}$ is the product of the fermion blocking kernels, and has a range

\footnotetext{
${ }^{24}$ Similar "tricks" have also been used recently in the applications of twisted-mass fermions [87], although in that case one does not face the issue of rooting.
} 
$\sim a_{c}$. It depends on the gauge fields on the fine lattice and all the blocked lattices (except the "last" one, $V_{n}$, although this will not matter here). In words, eq. (B.8) says that the blocked propagator is obtained from the original fine-lattice propagator by smearing over a range $a_{c}$ on both ends, and adding a contact term.

We next insert the result (B.8) into the correlator we are calculating, e.g. eq. (B.6), and integrate out all but the fine-lattice gauge fields. This brings us back to the original fine lattice action (that which is simulated), including the rooted determinant of $D_{\text {stag }}$. The integration also changes the combined kernel $\mathscr{Q}$, which depends on the blocked gauge fields. Consider the correlator (B.6), but with $|x-y| \gg a_{c}$, so that the contact term in $D_{n}^{-1}$ does not contribute, and the kernels $\mathscr{Q}$ at the two ends of $D_{n}^{-1}$ do not overlap. Then the gluon integration acts separately on the two ends, and changes the product of kernels into a smeared source:

$$
\mathscr{Q}^{\dagger}\left(x^{\prime \prime}, x\right)\left(\gamma_{5} \otimes \xi_{5}\right) \mathscr{Q}\left(x, x^{\prime}\right) \rightarrow \mathscr{S}_{5}\left(x^{\prime \prime}, x, x^{\prime}\right)
$$

Here $\mathscr{S}_{5}$ is a sum (weighted by phases) over products of links running from $x^{\prime \prime}$ to $x$ and then to $x^{\prime}$, possibly multiplied by nearby closed Wilson loops. It is no doubt horribly complicated, but we do not need its explicit form. Inserting this result into (B.6) we end up with

$$
C_{\pi}^{\mathrm{stag}}(x, y)=\left\langle\operatorname{tr}\left[\mathscr{S}_{5}\left(x^{\prime \prime}, x, x^{\prime}\right) D_{\text {stag }}^{-1}\left(x^{\prime}, y^{\prime}\right) \mathscr{S}_{5}^{\dagger}\left(y^{\prime}, y, y^{\prime \prime}\right) D_{\text {stag }}^{-1}\left(y^{\prime \prime}, x^{\prime \prime}\right)\right]\right\rangle_{\text {fine }}, \quad|x-y| \gg a_{c} .
$$

Thus we learn that the correlator we want to calculate, eq. (B.6), is, at long distances, equal to a correlator of the form that is actually calculated, i.e. involving $D_{\text {stag }}^{-1}$. This is an example of the "pull-back mapping" used extensively by Shamir.

We are not quite done. The result (B.10) involves the mesonic sources $\mathscr{S}_{5}$ rather than the sources used in practice. The former are smeared (size $\sim a_{c}$ ), complicated and not known explicitly, while the latter are typically ultralocal (size $\sim a_{f}$ ). In a physical theory this would not matter, at least for determining the states that are created. One need only ensure that the sources used in practice have the same transformation properties under the lattice symmetry group as the $\mathscr{S}_{5}$, properties which are determined by the operator that is inserted in the original coarse-lattice correlator [here $\left(\gamma_{5} \otimes \xi_{5}\right)$ ]. Using a spectral decomposition, this is sufficient to ensure that the actual correlator couples to the same states as contribute to (B.10).

This argument does not go through as stated with rooted staggered fermions-it is the theory after, not before, blocking which has been argued to be physical (up to $O\left(a_{f}\right)$ corrections). To make progress, I think one must rely on perturbation theory. Perturbation theory for rooted staggered fermions has been briefly discussed in secs. 5 and 8.1. It involves the replica trick for fermion loops, but keeps the full $D_{\text {stag }}^{-1}$ on valence lines. It is thus partially quenched, but in a controlled way since valence and sea propagators are the same (including the mass terms). Furthermore, by an extension of the argument in sec. 8.1, the results of this "rooted" PT become equal [up to errors of $O\left(a_{c}\right)$ ] to those in a continuum taste-invariant PQ theory, i.e. one with the correct number of sea quarks but with four times as many valence quarks. Using this perturbation theory, one can, in principle, relate the correlators involving ultralocal and smeared operators. As long as the quarks and gluons created by these operators have momenta satisfying $\left|p_{i}\right| \ll 1 / a_{c}$, then they will "see" the smeared operator as local. This, plus gauge invariance and lattice symmetries, implies that smeared and local operators are related by $Z$-factors independent of the external state. This holds 
up to corrections suppressed by $a_{c}|p|$, which, since the non-perturbative momenta of interest have $p \sim \Lambda_{\mathrm{QCD}}$, requires $a_{c} \Lambda_{\mathrm{QCD}} \ll 1$.

The upshot is that the non-perturbative long-distance part of the correlators which are actually calculated is proportional to that of the correlators involving smeared sources, such as (B.10), with the proportionality constant calculable in principle using PT. The latter correlators are themselves equal, up to $O\left(a_{f}\right)$ corrections, to correlators in a physical single-taste theory, such as (B.3). Thus the correlators actually calculated have no "valence rooting" problem in the continuum limit. ${ }^{25}$

Missing from this discussion is the issue of whether one can make use of the normalization of the correlators actually calculated. This is important because these normalizations are needed to determine matrix elements such as $f_{K}$ and $B_{K}$. In fact one can use the normalization. The two parts of the argument above-first, matching correlators to a theory with extra tastes and, second, using PT to match operators at the coarse and fine scales—can be combined into the single step of matching the original fine lattice correlator to one in a PQ continuum theory, using PT. What makes this possible is that taste is unbroken in rooted staggered perturbation theory (up to scaling violations), so the intermediate step of passing to the reweighted theory at scale $a_{c}$ is not needed. The direct "fine-lattice to PQ continuum" matching is what is used in practice in (rooted) staggered calculations of matrix elements.

\section{References}

[1] C. T. H. Davies et al. [HPQCD, UKQCD, MILC, and Fermilab Collaborations], Phys. Rev. Lett. 92, 022001 (2004) [arXiv:hep-lat/0304004].

[2] C. Aubin et al. [HPQCD, MILC, and UKQCD Collaborations], Phys. Rev. D 70, 031504 (2004) [arXiv:hep-lat/0405022].

[3] C. Aubin et al. [MILC Collaboration], Phys. Rev. D 70, 114501 (2004) [arXiv:hep-lat/0407028].

[4] C. Aubin et al. [Fermilab Lattice, MILC, and HPQCD Collaborations], Phys. Rev. Lett. 94, 011601 (2005) [arXiv:hep-ph/0408306].

[5] C. Aubin et al. [Fermilab Lattice, MILC, and HPQCD Collaborations], Phys. Rev. Lett. 95, 122002 (2005) [arXiv:hep-lat/0506030].

[6] A. S. Kronfeld [Fermilab Lattice Colaboration Collaboration], arXiv:hep-lat/0607011.

[7] W. Lee, "Progress in Kaon Physics on the Lattice", plenary talk at this conference, PoS(LAT2006)015 [arXiv:hep-lat/0610058].

[8] T. Onogi, "Heavy flavor physics from lattice QCD", plenary talk at this conference, PoS(LAT2006)017.

[9] K. Orginos, "Recent lattice QCD results on nucleon structure", plenary talk at this conference, PoS(LAT2006)018.

[10] S. Durr, PoS LAT2005, 021 (2006) [arXiv:hep-lat/0509026].

[11] C. Bernard et al., PoS LAT2005, 114 (2006) [arXiv:hep-lat/0509176].

\footnotetext{
${ }^{25}$ This assumes, of course, that the mass in the valence propagators is the same as that in the corresponding sea-quark rooted determinant. One can extend this set-up to partially quenched rooted staggered fermions, and use PQ rS $\chi \mathrm{PT}$ to control the unitarity violations.
} 
[12] A. Hasenfratz and R. Hoffmann, Phys. Rev. D 74, 014511 (2006) [arXiv:hep-lat/0604010], and arXiv:hep-lat/0609030.

[13] E. Follana, A. Hart and C. T. H. Davies, Nucl. Phys. Proc. Suppl. 153, 106 (2006).

[14] C. DeTar, E. Gregory, and A. Hart, talks at this conference.

[15] C. Bernard, M. Golterman and Y. Shamir, Phys. Rev. D 73, 114511 (2006) [arXiv:hep-lat/0604017].

[16] Y. Shamir, arXiv:hep-lat/0607007.

[17] C. Bernard, Phys. Rev. D 73, 114503 (2006) [arXiv:hep-lat/0603011].

[18] M. Creutz, arXiv:hep-lat/0603020.

[19] M. Creutz, arXiv:hep-lat/0608020.

[20] C. Bernard, M. Golterman, Y. Shamir and S. R. Sharpe, arXiv:hep-lat/0603027.

[21] S. Dürr and C. Hoelbling, Phys. Rev. D 74, 014513 (2006) [arXiv:hep-lat/0604005].

[22] M. Golterman, Y. Shamir and B. Svetitsky, arXiv:hep-lat/0602026, Phys. Rev. D in press.

[23] L. Susskind, Phys. Rev. D 16, 3031 (1977);

[24] F. Gliozzi, Nucl. Phys. B 204, 419 (1982).

[25] H. Kluberg-Stern, A. Morel, O. Napoly and B. Petersson, Nucl. Phys. B 220, 447 (1983).

[26] M. F. L. Golterman and J. Smit, Nucl. Phys. B 245, 61 (1984); M. F. L. Golterman, Nucl. Phys. B 273, 663 (1986).

[27] P. Becher and H. Joos, Z. Phys. C 15, 343 (1982).

[28] P. Mitra, Phys. Lett. B 123, 77 (1983).

[29] M. F. L. Golterman and J. Smit, Phys. Lett. B 140, 392 (1984).

[30] M. Gockeler, Phys. Lett. B 142, 197 (1984).

[31] P. Mitra and P. Weisz, Phys. Lett. B 126, 355 (1983).

[32] E. Marinari, G. Parisi and C. Rebbi, Nucl. Phys. B 190, 734 (1981).

[33] K. Jansen, Nucl. Phys. Proc. Suppl. 129, 3 (2004) [arXiv:hep-lat/0311039].

[34] B. Bunk, M. Della Morte, K. Jansen and F. Knechtli, Nucl. Phys. B 697, 343 (2004) [arXiv:hep-lat/0403022].

[35] D. H. Adams, Phys. Rev. D 72, 114512 (2005) [arXiv:hep-lat/0411030].

[36] Y. Shamir, Phys. Rev. D 71, 034509 (2005) [arXiv:hep-lat/0412014].

[37] L. Giusti, "Light dynamical fermions on the lattice: toward the chiral regime of QCD", plenary talk at this conference, PoS(LAT2006)009.

[38] K. Orginos, D. Toussaint and R. L. Sugar [MILC Collaboration], Phys. Rev. D 60, 054503 (1999) [arXiv:hep-lat/9903032].

[39] K. Jansen, "Status report on ILDG activities", plenary talk at this conference, PoS(LAT2006)013 [arXiv:hep-lat/0609012].

[40] C. Bernard et al. [MILC Collaboration], arXiv:hep-lat/0609053. 
[41] S. R. Sharpe, Phys. Rev. D 56, 7052 (1997) [Erratum-ibid. D 62, 099901 (2000)] [arXiv:hep-lat/9707018].

[42] Q. Mason, H. D. Trottier, R. Horgan, C. T. H. Davies and G. P. Lepage [HPQCD Collaboration], Phys. Rev. D 73, 114501 (2006) [arXiv:hep-ph/0511160].

[43] M. Gockeler et al., Phys. Rev. D 73, 054508 (2006) [arXiv:hep-lat/0601004].

[44] M.E. Fisher, S-K. Ma and B.G. Nickel, Phys. Rev. Lett. 29, 917 (1972).

[45] M. Suzuki, Y. Yamazaki and G. Igarashi, Phys. Lett. 42A, 313 (1972).

[46] D. Dantchev and J. Rudnick, Eur. Phys. J. B 21, 251 (2001).

[47] M. Aizenman and R. Fernández, Lett. Math. Phys. 16, 39 (1988).

[48] E. Luitjen and H.W.J. Blöte, Phys. Rev. B 56, 8945 (1997).

[49] E. Luitjen and H.W.J. Blöte, Phys. Rev. Lett. 89, 025703 (2002).

[50] C. W. Bernard and M. Golterman, Phys. Rev. D 49, 486 (1994) [arXiv:hep-lat/9306005].

[51] J. Giedt, arXiv:hep-lat/0606003.

[52] Q. Mason et al. [HPQCD Collaboration], Phys. Rev. Lett. 95, 052002 (2005) [arXiv:hep-lat/0503005].

[53] M. Della Morte, R. Frezzotti, J. Heitger, J. Rolf, R. Sommer and U. Wolff [ALPHA Collaboration], Nucl. Phys. B 713, 378 (2005) [arXiv:hep-lat/0411025].

[54] M. Luscher, R. Narayanan, P. Weisz and U. Wolff, Nucl. Phys. B 384, 168 (1992) [arXiv:hep-lat/9207009].

[55] M. Golterman and Y. Shamir, Phys. Rev. D 68, 074501 (2003) [arXiv:hep-lat/0306002] and Nucl. Phys. Proc. Suppl. 129, 149 (2004) [arXiv:hep-lat/0309027]; M. Golterman, Y. Shamir and B. Svetitsky, Phys. Rev. D 71, 071502 (2005) [arXiv:hep-lat/0407021] and Phys. Rev. D 72, 034501 (2005) [arXiv:hep-lat/0503037].

[56] C. Bernard, M. Golterman and Y. Shamir, talks at this conference, arXiv:hep-lat/0610003.

[57] G. P. Lepage, Nucl. Phys. Proc. Suppl. 60A, 267 (1998) [arXiv:hep-lat/9707026] and Phys. Rev. D 59, 074502 (1999) [arXiv:hep-lat/9809157].

[58] J. F. Lagae and D. K. Sinclair, Nucl. Phys. Proc. Suppl. 63, 892 (1998) [arXiv:hep-lat/9709035].

[59] W. J. Lee and S. R. Sharpe, Phys. Rev. D 60, 114503 (1999) [arXiv:hep-lat/9905023].

[60] Y. b. Luo, Phys. Rev. D 55, 353 (1997) [arXiv:hep-lat/9604025].

[61] S. R. Sharpe, Nucl. Phys. Proc. Suppl. 34, 403 (1994) [arXiv:hep-lat/9312009] and Proc. TASI 94 (World Scientific, 1995) [arXiv:hep-ph/9412243].

[62] C. Bernard [MILC Collaboration], Phys. Rev. D 65, 054031 (2002) [arXiv:hep-lat/0111051]; C. Aubin and C. Bernard, Phys. Rev. D 68, 034014 (2003) [arXiv:hep-lat/0304014] and Phys. Rev. D 68, 074011 (2003) [arXiv:hep-lat/0306026].

[63] S. R. Sharpe and R. L. Singleton, Phys. Rev. D 58, 074501 (1998) [arXiv:hep-lat/9804028].

[64] K. Symanzik, Commun. Math. Phys. 45, 79 (1975), Nucl. Phys. B 226, 187 (1983) and Nucl. Phys. B 226, 205 (1983).

[65] S. Weinberg, PhysicaA 96, 327 (1979). 
[66] H. Leutwyler, Annals Phys. 235, 165 (1994) [arXiv:hep-ph/9311274].

[67] S. R. Sharpe and N. Shoresh, Phys. Rev. D 64, 114510 (2001) [arXiv:hep-lat/0108003].

[68] S. R. Sharpe, arXiv:hep-lat/0607016.

[69] M. Golterman, Y. Shamir and B. Svetitsky, talk at this conference, arXiv:hep-lat/0609051.

[70] S. Dürr and C. Hoelbling, Phys. Rev. D 69, 034503 (2004) [arXiv:hep-lat/0311002] and Phys. Rev. D 71, 054501 (2005) [arXiv:hep-lat/0411022];

E. Follana, A. Hart and C. T. H. Davies [HPQCD Collaboration], Phys. Rev. Lett. 93, 241601 (2004) [arXiv:hep-lat/0406010];

S. Dürr, C. Hoelbling and U. Wenger, Phys. Rev. D 70, 094502 (2004) [arXiv:hep-lat/0406027].

[71] J. Smit and J. C. Vink, Nucl. Phys. B 286, 485 (1987).

[72] C. Bernard, Phys. Rev. D 71, 094020 (2005) [arXiv:hep-lat/0412030].

[73] M. Creutz, arXiv:hep-ph/9608216.

[74] C. Aubin and Q. h. Wang, Phys. Rev. D 70, 114504 (2004) [arXiv:hep-lat/0410020].

[75] R. F. Dashen, Phys. Rev. D 3, 1879 (1971);

E. Witten, Annals Phys. 128, 363 (1980).

[76] O. Bar, C. Bernard, G. Rupak and N. Shoresh, Phys. Rev. D 72, 054502 (2005) [arXiv:hep-lat/0503009].

[77] U. Heller, "Recent progress in finite temperature lattice QCD", plenary talk at this conference, PoS(LAT2006)011.

[78] C. Schmidt, "Lattice QCD at finite density", plenary talk at this conference, PoS(LAT2006)021.

[79] K. Splittorff, "The sign problem in the epsilon-regime of QCD”, plenary talk at this conference, PoS(LAT2006)023 [arXiv:hep-lat/0610072].

[80] M. Stephanov, "QCD phase diagram: an overview”, plenary talk at this conference, PoS(LAT2006)024.

[81] T. DeGrand, "The one-flavor quark condensate and related problems," plenary talk at this conference, PoS(LAT2006)005, [arXiv:hep-lat/0609031].

[82] H. S. Sharatchandra, H. J. Thun and P. Weisz, Nucl. Phys. B 192, 205 (1981).

[83] D. Daniel and S. N. Sheard, Nucl. Phys. B 302, 471 (1988).

[84] D. Daniel and S. N. Sheard, Nucl. Phys. B 302, 471 (1988).

[85] S. R. Sharpe, "Staggered fermions on the lattice", unpublished preprint, DOE/ER/40614-5.

[86] S. R. Sharpe, A. Patel, R. Gupta, G. Guralnik and G. W. Kilcup, Nucl. Phys. B 286, 253 (1987);

G. Kilcup, R. Gupta and S. R. Sharpe, Phys. Rev. D 57, 1654 (1998) [arXiv:hep-lat/9707006].

[87] R. Frezzotti and G. C. Rossi, JHEP 0410, 070 (2004) [arXiv:hep-lat/0407002]. 